\title{
Aliphatic C-H activation in the formation of abnormal carbenes with iridium: a combined experimental and theoretical study
}

\author{
Mónica Viciano, Marta Feliz, Rosa Corberán, Jose A. Mata, Eric Clot, Eduardo Peris
}

\section{Supporting Information}

Complete list of authors for reference 36:

Gaussian 03, Revision C.02, M. J. Frisch, G. W. Trucks, H. B. Schlegel, G. E. Scuseria, M. A. Robb, J. R. Cheeseman, J. A. Montgomery, Jr., T. Vreven, K. N. Kudin, J. C. Burant, J. M. Millam, S. S. Iyengar, J. Tomasi, V. Barone, B. Mennucci, M. Cossi, G. Scalmani, N. Rega, G. A. Petersson, H. Nakatsuji, M. Hada, M. Ehara, K. Toyota, R. Fukuda, J. Hasegawa, M. Ishida, T. Nakajima, Y. Honda, O. Kitao, H. Nakai, M. Klene, X. Li, J. E. Knox, H. P. Hratchian, J. B. Cross, C. Adamo, J. Jaramillo, R. Gomperts, R. E. Stratmann, O. Yazyev, A. J. Austin, R. Cammi, C. Pomelli, J. W. Ochterski, P. Y. Ayala, K. Morokuma, G. A. Voth, P. Salvador, J. J. Dannenberg, V. G. Zakrzewski, S. Dapprich, A. D. Daniels, M. C. Strain, O. Farkas, D. K. Malick, A. D. Rabuck, K. Raghavachari, J. B. Foresman, J. V. Ortiz, Q. Cui, A. G. Baboul, S. Clifford, J. Cioslowski, B. B. Stefanov, G. Liu, A. Liashenko, P. Piskorz, I. Komaromi, R. L. Martin, D. J. Fox, T. Keith, M. A. Al-Laham, C. Y. Peng, A. Nanayakkara, M. Challacombe, P. M. W. Gill, B. Johnson, W. Chen, M. W. Wong, C. Gonzalez, and J. A. Pople, Gaussian, Inc., Wallingford CT, 2004. 


\section{Cartesian coordinates for the molecules optimized in gas phase at the B3PW91 level and B3PW91/PCM(acetonitrile) energy (a.u.) on the gas phase optimized geometry}

$\mathrm{Cp}^{*} \operatorname{IrI}_{2}: \mathrm{E}=-517.455597657$

$\begin{array}{lrrr}\text { Ir } & 0.19875 & -0.01227 & -0.09531 \\ \text { I } & -0.67847 & 1.00878 & 2.17593 \\ \text { C } & 2.18957 & 0.85403 & -0.19627 \\ \text { C } & 2.26457 & -0.50299 & 0.25531 \\ \text { C } & 1.70497 & -1.35964 & -0.7913 \\ \text { C } & 1.31433 & -0.53074 & -1.89903 \\ \text { C } & 1.54713 & 0.84221 & -1.50357 \\ \mathrm{C} & 2.70655 & 2.05635 & 0.52252 \\ \mathrm{H} & 2.58387 & 1.95587 & 1.60304 \\ \mathrm{H} & 2.17952 & 2.96214 & 0.21621 \\ \mathrm{H} & 3.77312 & 2.19426 & 0.30576 \\ \mathrm{C} & 2.86596 & -0.97576 & 1.53731 \\ \mathrm{H} & 3.93414 & -1.18451 & 1.39751 \\ \mathrm{H} & 2.38602 & -1.89177 & 1.88821 \\ \mathrm{H} & 2.76131 & -0.2249 & 2.32242 \\ \mathrm{C} & 1.63933 & -2.85026 & -0.73973 \\ \mathrm{H} & 1.43848 & -3.20373 & 0.27402 \\ \mathrm{H} & 2.59564 & -3.27903 & -1.06522 \\ \mathrm{H} & 0.85172 & -3.2351 & -1.3896 \\ \mathrm{C} & 0.78356 & -0.99603 & -3.21418 \\ \mathrm{H} & 0.11581 & -0.25534 & -3.65866 \\ \mathrm{H} & 0.21396 & -1.92142 & -3.10881 \\ \mathrm{H} & 1.61031 & -1.17662 & -3.91225 \\ \mathrm{C} & 1.27055 & 2.04706 & -2.34023 \\ \mathrm{H} & 2.15272 & 2.29189 & -2.9454 \\ \mathrm{H} & 1.03266 & 2.91418 & -1.72061 \\ \mathrm{H} & 0.43027 & 1.87607 & -3.01564 \\ \mathrm{I} & -2.20987 & -0.78959 & -0.84833\end{array}$

bisIm $^{2+}: \mathrm{E}=-648.482992762$

$\mathrm{N}, 0,-3.0915500879,-0.3349205597,-0.5035784452$ C, $0,-1.1668168996,-1.408786029,-0.4152908025$ $\mathrm{N}, 0,-1.1129582875,-0.2749055151,0.3799149181$ $\mathrm{C}, 0,-2.4030220694,-1.4344911001,-0.9733183086$ $\mathrm{N}, 0,1.2904296895,-0.2027455161,0.7238433952$ C, $0,-4.4769617664,-0.0011270007,-0.8642403224$ $\mathrm{H}, 0,-5.0890150862,0.0764077536,0.0355896477$ $\mathrm{H}, 0,-4.8678083246,-0.8035761724,-1.4882130182$ $\mathrm{H}, \mathrm{O},-4.5044882474,0.933316824,-1.4279938518$ C, $0,-2.3061668984,0.3693808488,0.324037683$ $\mathrm{N}, 0,3.1822396598,0.0050010675,-0.3115491594$ C, $0,-2.6997716107,1.5892962573,1.0727622863$ $\mathrm{H}, \mathrm{O},-3.5728678875,2.0542684933,0.6123231905$ $\mathrm{H}, \mathrm{O},-1.9031585551,2.3381203625,1.0819909045$ $\mathrm{H}, \mathrm{O},-2.9582519494,1.3507590867,2.1113604663$ C, $0,-0.0173234519,0.0725478538,1.2899521574$ C, $0,2.0465481449,-1.3280334187,1.0281185887$ $\mathrm{H}, 0,1.6927572254,-2.1034771153,1.6912583908$ C, $0,3.226672963,-1.1935321771,0.3745252518$ $\mathrm{H}, \mathrm{O}, 4.0912575416,-1.8398297596,0.3470121672$ C, $0,4.2725899757,0.5384848703,-1.139732788$ $\mathrm{H}, 0,3.9820457007,0.5296477066,-2.1924653535$ $\mathrm{H}, 0,5.1467410915,-0.0968980017,-1.0047915598$ $\mathrm{H}, 0,4.5195740795,1.5523362526,-0.8215965485$ C, $0,2.005931181,0.6069104559,-0.0982017558$ C, $0,1.5983748168,1.928728063,-0.6321519646$ $\mathrm{H}, \mathrm{O}, 0.5127283816,2.0287961164,-0.6671892422$ $\mathrm{H}, 0,1.9731035577,2.0638761834,-1.6499345461$ $\mathrm{H}, 0,2.0039049054,2.7415454443,-0.0171025488$
$\mathrm{H}, 0,-0.3360904944,-2.0876432987,-0.525930444$ $\mathrm{H}, 0,-2.8461316321,-2.1387761669,-1.6613362549$ $\mathrm{H}, 0,-0.0962833218,1.1287813149,1.5479735427$ $\mathrm{H}, \mathrm{O},-0.1054701127,-0.5109331012,2.2098699446$

$$
\mathrm{AcO}^{-}: \mathrm{E}=-228.511565382
$$

$C, 0,0.1728364294,-0.000009587,0.1351032159$ $0,0,-0.0578375241,-0.0000432812,1.368214545$ $0,0,1.2679184457,-0.0000053971,-0.4797352066$ C, $0,-1.0911561156,0.0000437411,-0.7967812333$ $\mathrm{H}, 0,-1.0712853007,0.8793555664,-1.455271057$ $\mathrm{H}, 0,-1.0712739703,-0.8791688435,-1.4554030877$ $\mathrm{H}, 0,-2.0281699844,-0.0000022216,-0.2270924583$

\section{$\mathrm{AcOH}: \mathrm{E}=-229.007227008$}

C, $0,0.0169915112,-0.0000081832,0.1533384056$ $0,0,-0.0516649817,-0.0000651655,1.3607440177$ $0,0,1.1972470606,0.0000104553,-0.5065472439$ $\mathrm{H}, \mathrm{O}, 1.8826641308,-0.0000286088,0.179751949$ C, $0,-1.145887744,0.0000516881,-0.8002998043$ $\mathrm{H}, \mathrm{O},-1.0978570775,0.8807610745,-1.4469305629$ $\mathrm{H}, \mathrm{O},-1.0978658451,-0.8805794561,-1.4470374093$ $\mathrm{H}, \mathrm{O},-2.0782204422,0.0000236331,-0.2375897751$

$\left[\mathrm{Cp}^{*} \operatorname{IrI}_{2}\right]_{2}: \mathrm{E}=-1034.92228010$

$\begin{array}{llll}\text { Ir } & -3.612703 & 6.118675 & -9.195433 \\ \text { I } & -3.850442 & 3.747623 & -7.834672 \\ \text { C } & -4.730083 & 5.374904 & -11.000893 \\ \text { C } & -5.139909 & 6.729924 & -10.777218 \\ \text { C } & -3.964691 & 7.586235 & -10.824539 \\ \text { C } & -2.838205 & 6.746538 & -11.146663 \\ \text { C } & -3.297526 & 5.370137 & -11.227833 \\ \text { C } & -5.637396 & 4.197883 & -11.113653 \\ \text { H } & -6.496823 & 4.285621 & -10.446821 \\ \text { H } & -5.122168 & 3.265078 & -10.881801 \\ \text { H } & -6.012626 & 4.134825 & -12.142802 \\ \mathrm{C} & -6.548757 & 7.196513 & -10.660411 \\ \mathrm{H} & -6.937482 & 7.350778 & -11.675982 \\ \mathrm{H} & -6.627764 & 8.141060 & -10.121584 \\ \mathrm{H} & -7.188137 & 6.462115 & -10.167947 \\ \mathrm{C} & -3.977521 & 9.077344 & -10.757175 \\ \mathrm{H} & -4.678803 & 9.435943 & -10.000289 \\ \mathrm{H} & -4.284169 & 9.490564 & -11.725575 \\ \mathrm{H} & -2.990071 & 9.480682 & -10.523934 \\ \mathrm{C} & -1.461508 & 7.227996 & -11.465237 \\ \mathrm{H} & -0.704018 & 6.462568 & -11.282958 \\ \mathrm{H} & -1.200423 & 8.128197 & -10.904327 \\ \mathrm{H} & -1.411095 & 7.485023 & -12.530061 \\ \mathrm{C} & -2.506762 & 4.184693 & -11.675711 \\ \mathrm{H} & -2.693619 & 3.999029 & -12.739948 \\ \mathrm{H} & -2.788519 & 3.284874 & -11.123948 \\ \mathrm{H} & -1.432837 & 4.340980 & -11.554896 \\ \mathrm{I} & -5.233897 & 7.380537 & -7.367488 \\ \mathrm{Ir} & -2.786387 & 5.956638 & -6.145893 \\ \mathrm{I} & -1.371966 & 4.646589 & -8.098650 \\ \mathrm{C} & -1.173254 & 5.715009 & -4.596372 \\ \mathrm{C} & -1.721766 & 7.029333 & -4.435898 \\ \mathrm{C} & -3.147786 & 6.910459 & -4.173252 \\ \mathrm{C} & -3.453377 & 5.503247 & -4.108493 \\ \mathrm{C} & -2.242230 & 4.754635 & -4.399606 \\ & & & \end{array}$




$\begin{array}{lrll}\mathrm{C} & 0.267707 & 5.391047 & -4.796351 \\ \mathrm{H} & 0.770922 & 6.146887 & -5.401708 \\ \mathrm{H} & 0.402272 & 4.423524 & -5.281255 \\ \mathrm{H} & 0.763760 & 5.355499 & -3.818297 \\ \mathrm{C} & -0.935940 & 8.293240 & -4.401007 \\ \mathrm{H} & -0.564667 & 8.430517 & -3.376507 \\ \mathrm{H} & -1.540399 & 9.162776 & -4.660395 \\ \mathrm{H} & -0.071508 & 8.261247 & -5.066140 \\ \mathrm{C} & -4.061482 & 8.040084 & -3.830882 \\ \mathrm{H} & -3.861072 & 8.916637 & -4.451168 \\ \mathrm{H} & -3.919893 & 8.327966 & -2.782235 \\ \mathrm{H} & -5.110457 & 7.765583 & -3.959100 \\ \mathrm{C} & -4.761860 & 4.918961 & -3.690081 \\ \mathrm{H} & -4.909197 & 3.911391 & -4.085037 \\ \mathrm{H} & -5.605974 & 5.545132 & -3.987619 \\ \mathrm{H} & -4.785364 & 4.843664 & -2.596357 \\ \mathrm{C} & -2.055948 & 3.275702 & -4.303508 \\ \mathrm{H} & -1.624946 & 3.022185 & -3.327827 \\ \mathrm{H} & -1.376672 & 2.907567 & -5.075884 \\ \mathrm{H} & -3.002386 & 2.738375 & -4.392234 \\ \mathrm{I} & -2.600721 & 8.321491 & -7.536917\end{array}$

TS1me : $\mathrm{E}=-1165.88823278$

$\begin{array}{lrcc}\mathrm{C} & -0.33144 & 3.13098 & -3.02783 \\ \mathrm{H} & -1.03272 & 3.48958 & -2.27095 \\ \mathrm{H} & -0.63809 & 3.5442 & -3.99623 \\ \mathrm{H} & 0.65601 & 3.53432 & -2.79459 \\ \mathrm{C} & 2.18457 & 1.28164 & -3.7359 \\ \mathrm{H} & 2.94206 & 0.51621 & -3.55362 \\ \mathrm{H} & 2.44566 & 2.18184 & -3.17499 \\ \mathrm{H} & 2.23498 & 1.53866 & -4.80072 \\ \mathrm{C} & 1.13932 & -1.76167 & -3.94637 \\ \mathrm{H} & 0.95246 & -1.94733 & -5.01061 \\ \mathrm{H} & 0.85756 & -2.66149 & -3.39461 \\ \mathrm{H} & 2.21324 & -1.60538 & -3.82555 \\ \mathrm{H} & 2.54307 & -1.01769 & 4.07468 \\ \mathrm{H} & 0.29344 & 2.84326 & 5.57401 \\ \mathrm{H} & 0.47075 & 1.73942 & 6.94109 \\ \mathrm{H} & 1.72043 & 1.79565 & 5.70505 \\ \mathrm{H} & 1.54417 & -0.24465 & -1.16137 \\ \mathrm{H} & 2.69736 & 1.17553 & -0.7225 \\ \mathrm{H} & 1.31307 & 1.73233 & 0.21082 \\ \mathrm{I} & -1.58782 & 1.43417 & 0.36185 \\ & & & \end{array}$

TS1vy $: E=-1165.88422835$

\begin{tabular}{|c|c|c|c|}
\hline 77 & 1.086657 & -0.303688 & 0.283198 \\
\hline & 2.842826 & 1.676979 & -0.373140 \\
\hline 7 & -1.758118 & 1.057723 & 0.973523 \\
\hline 6 & 2.139055 & -1.403726 & 1.895717 \\
\hline 6 & 2.913758 & -1.613248 & 0.700411 \\
\hline 6 & 2.061531 & -2.222950 & -0.279676 \\
\hline 6 & 2.675196 & -0.876842 & 3.187895 \\
\hline 1 & 1.881274 & -0.518306 & 3.846678 \\
\hline 1 & 3.376315 & -0.056047 & 3.023459 \\
\hline 1 & 3.211166 & -1.673343 & 3.718057 \\
\hline 6 & 4.366848 & -1.338897 & 0.539084 \\
\hline 1 & 4.631420 & -1.125673 & -0.497005 \\
\hline 1 & 4.913071 & -2.239704 & 0.850213 \\
\hline 1 & 4.704490 & -0.506557 & 1.157314 \\
\hline 6 & 2.500849 & -2.686882 & -1.628854 \\
\hline 1 & 3.032950 & -3.640472 & -1.524433 \\
\hline 1 & 3.179435 & -1.971316 & -2.097531 \\
\hline 1 & 1.655788 & -2.837019 & -2.301335 \\
\hline 6 & -0.366133 & 1.241635 & 0.767945 \\
\hline 6 & -2.384189 & 2.257735 & 1.045186 \\
\hline 6 & -2.404552 & -0.241866 & 1.009765 \\
\hline 1 & -2.714707 & -0.493451 & 2.025422 \\
\hline 1 & -1.640280 & -0.947015 & 0.668048 \\
\hline 7 & -3.575615 & -0.336885 & 0.140439 \\
\hline 6 & 0.836101 & -2.029305 & 1.695125 \\
\hline 6 & 0.781301 & -2.531135 & 0.356547 \\
\hline 6 & -0.150907 & -2.2733 & 2.791767 \\
\hline 1 & -1.125330 & -2.586177 & 2.411618 \\
\hline 1 & 0.218434 & -3.088045 & 3.426072 \\
\hline 1 & -0.278654 & -1.401846 & 3.439625 \\
\hline 6 & -0.282321 & -3.376909 & -0.259923 \\
\hline 1 & 0.066408 & -4.414411 & -0.322621 \\
\hline 1 & -1.201326 & -3.380658 & 0.331671 \\
\hline 1 & -0.517714 & -3.040563 & -1.273835 \\
\hline 6 & -3.581107 & -0.038842 & -1.212116 \\
\hline 6 & -4.784645 & -0.835383 & 0.499204 \\
\hline 7 & -1.449023 & 3.201461 & 0.884771 \\
\hline 7 & -5.545684 & -0.845808 & -0.605128 \\
\hline 6 & -0.220631 & 2.609993 & 0.736076 \\
\hline 6 & -4.818724 & -0.352897 & -1.671710 \\
\hline 1 & 0.687104 & 3.182614 & 0.617068 \\
\hline 1 & -5.237098 & -0.278091 & -2.663937 \\
\hline
\end{tabular}




$\begin{array}{rrrr}6 & -6.932569 & -1.318123 & -0.687426 \\ 1 & -6.994091 & -2.361164 & -0.372636 \\ 1 & -7.583007 & -0.696284 & -0.069129 \\ 1 & -7.255850 & -1.243416 & -1.724702 \\ 6 & -1.676972 & 4.650075 & 0.884436 \\ 1 & -2.433777 & 4.911398 & 0.143491 \\ 1 & -1.983768 & 4.985329 & 1.877562 \\ 1 & -0.740826 & 5.138317 & 0.617662 \\ 6 & -5.199624 & -1.328845 & 1.837275 \\ 1 & -4.850062 & -2.353595 & 2.008930 \\ 1 & -4.815989 & -0.699358 & 2.644110 \\ 1 & -6.287740 & -1.333802 & 1.920314 \\ 6 & -3.829745 & 2.516624 & 1.261060 \\ 1 & -4.394851 & 2.467546 & 0.323271 \\ 1 & -4.259189 & 1.802522 & 1.966978 \\ 1 & -3.972469 & 3.513551 & 1.682497 \\ 1 & 0.584265 & 0.720325 & 1.510915 \\ 1 & -2.686885 & 0.314861 & -1.714295 \\ 53 & 0.017605 & -0.075904 & -2.230969\end{array}$

$\mathbf{I r I}_{2} \mathbf{m e H}^{+}: \mathrm{E}=-1165.90322496$

$\begin{array}{lll}3.64584 & -2.30233 & -2.99461 \\ 2.47659 & -1.07931 & -3.49704 \\ 3.69986 & 1.2461 & -2.2695 \\ 3.5809 & 2.28106 & -1.94205 \\ 3.10627 & 1.09289 & -3.17334 \\ 4.75201 & 1.11043 & -2.54662 \\ 4.12391 & 1.67223 & 0.90083 \\ 5.19265 & 1.50689 & 1.0812 \\ 3.64917 & 1.86691 & 1.86501 \\ 4.02757 & 2.56472 & 0.27998 \\ -3.98885 & 2.70587 & -0.51012 \\ -5.55663 & -1.33272 & -2.50882 \\ -6.95352 & -0.61806 & -1.69933 \\ -5.74843 & 0.43062 & -2.43594 \\ 1.5511 & 1.59567 & -0.05555 \\ 0.79867 & 1.48662 & -2.36638 \\ -0.27167 & 0.09433 & -2.29013 \\ -0.37529 & -2.05296 & -0.22268\end{array}$

$\operatorname{IrI}_{2} \mathbf{v y H}^{+}: \mathrm{E}=-1165.89685033$

$\begin{array}{lccc}77 & 1.19312 & -0.01047 & 0.3113 \\ 53 & 2.22365 & 1.00344 & -2.01873 \\ 7 & -1.70273 & 1.28942 & 0.73788 \\ 6 & 2.85621 & 0.05469 & 1.80493 \\ 6 & 3.18672 & -1.03511 & 0.90422 \\ 6 & 2.15852 & -2.00886 & 1.00808 \\ 6 & 3.77484 & 1.18028 & 2.15153 \\ 1 & 3.24379 & 2.03091 & 2.58242 \\ 1 & 4.34256 & 1.52393 & 1.28455 \\ 1 & 4.49493 & 0.82399 & 2.89841 \\ 6 & 4.44268 & -1.15515 & 0.10952 \\ 1 & 4.29791 & -1.77107 & -0.77926 \\ 1 & 5.21767 & -1.6261 & 0.72685 \\ 1 & 4.81288 & -0.1818 & -0.21624 \\ 6 & 2.17853 & -3.35881 & 0.38029 \\ 1 & 2.77924 & -4.02553 & 1.01267 \\ 1 & 2.63413 & -3.34041 & -0.61119 \\ 1 & 1.17923 & -3.78519 & 0.28852 \\ 6 & -0.34185 & 1.38297 & 0.35807 \\ 6 & -2.29695 & 2.5153 & 0.7399 \\ 6 & -2.41066 & 0.07465 & 1.0646 \\ 1 & -2.80514 & 0.11449 & 2.08267 \\ 1 & -1.6967 & -0.74678 & 0.95947 \\ 7 & -3.53889 & -0.16263 & 0.15372 \\ 6 & 1.60612 & -0.26641 & 2.47611 \\ 6 & 1.16143 & -1.53289 & 1.97071 \\ 6 & 1.0116 & 0.5105 & 3.60447 \\ 1 & -0.05643 & 0.31336 & 3.71419 \\ 1 & 1.49843 & 0.22469 & 4.54433 \\ 1 & 1.14987 & 1.58556 & 3.47312 \\ 6 & 0.03965 & -2.36671 & 2.49247 \\ 1 & 0.44296 & -3.15301 & 3.14177 \\ 1 & -0.66054 & -1.78117 & 3.092 \\ 1 & -0.5007 & -2.85858 & 1.67793 \\ 6 & -3.54198 & 0.10488 & -1.20325 \\ 6 & -4.68583 & -0.79613 & 0.48928 \\ 7 & -1.37634 & 3.39069 & 0.34022 \\ 7 & -5.41306 & -0.91627 & -0.63229 \\ 6 & -0.18963 & 2.72542 & 0.10881 \\ 6 & -4.72289 & -0.35724 & -1.68891 \\ 1 & 0.67756 & 3.25842 & -0.24963 \\ 1 & -5.12066 & -0.3462 & -2.69197 \\ 6 & -6.73341 & -1.54592 & -0.73799 \\ 1 & -6.6807 & -2.58686 & -0.41467\end{array}$




$\begin{array}{llcr}1 & -7.46478 & -0.99851 & -0.14033 \\ 1 & -7.04013 & -1.51807 & -1.78261 \\ 6 & -1.56883 & 4.83331 & 0.18374 \\ 1 & -2.44656 & 5.03362 & -0.43235 \\ 1 & -1.67762 & 5.31106 & 1.16011 \\ 1 & -0.6911 & 5.24144 & -0.31559 \\ 6 & -5.06465 & -1.29627 & 1.83465 \\ 1 & -4.47762 & -2.17948 & 2.10991 \\ 1 & -4.91732 & -0.5331 & 2.60473 \\ 1 & -6.11778 & -1.57877 & 1.85549 \\ 6 & -3.69878 & 2.8335 & 1.11226 \\ 1 & -4.3898 & 2.68214 & 0.27504 \\ 1 & -4.03614 & 2.21995 & 1.95188 \\ 1 & -3.7789 & 3.87649 & 1.42462 \\ 1 & 1.88488 & 1.39511 & 0.39122 \\ 1 & -2.70643 & 0.56971 & -1.70179 \\ 53 & -0.37478 & -1.58028 & -1.32211\end{array}$

IrI $_{2} \mathbf{m e}: \mathrm{E}=-1165.49059693$

$\begin{array}{lcrc}\text { Ir } & 1.28154 & -0.24951 & -0.16761 \\ \mathrm{~N} & -0.20824 & 3.24404 & -1.15952 \\ \mathrm{C} & -2.40353 & 3.23856 & -0.98555 \\ \mathrm{I} & 0.24738 & 1.37422 & 1.83846 \\ \mathrm{~N} & -1.94469 & 1.96293 & -1.30707 \\ \mathrm{C} & -1.3122 & 4.02655 & -0.88177 \\ \mathrm{H} & -1.22171 & 5.07146 & -0.63152 \\ \mathrm{~N} & -3.56652 & 0.42386 & -0.41183 \\ \mathrm{C} & 1.17351 & 3.69083 & -1.11114 \\ \mathrm{H} & 1.72883 & 3.03424 & -0.43734 \\ \mathrm{H} & 1.19628 & 4.70888 & -0.724 \\ \mathrm{H} & 1.61379 & 3.67603 & -2.11213 \\ \mathrm{C} & -0.57993 & 1.97213 & -1.41427 \\ \mathrm{~N} & -4.91482 & -0.77213 & 0.78353 \\ \mathrm{C} & 0.31994 & 0.88718 & -1.76237 \\ \mathrm{C} & -2.7965 & 0.84633 & -1.59084 \\ \mathrm{C} & -3.29978 & 0.72744 & 0.90585 \\ \mathrm{H} & -2.47977 & 1.36879 & 1.20407 \\ \mathrm{C} & -4.15973 & -0.01226 & 1.65236 \\ \mathrm{H} & -4.27479 & -0.07998 & 2.72212 \\ \mathrm{C} & -5.93558 & -1.73616 & 1.18541 \\ \mathrm{H} & -6.89445 & -1.49216 & 0.72419 \\ \mathrm{H} & -6.04394 & -1.68463 & 2.26787 \\ \mathrm{H} & -5.6291 & -2.74699 & 0.90935 \\ \mathrm{C} & -4.53367 & -0.51164 & -0.4764 \\ \mathrm{C} & -5.04108 & -1.15321 & -1.71367 \\ \mathrm{H} & -5.42519 & -0.41214 & -2.42242 \\ \mathrm{H} & -5.85018 & -1.84737 & -1.48562 \\ \mathrm{C} & 3.37916 & 0.23528 & 0.10748 \\ \mathrm{C} & 3.04633 & -0.79311 & 1.0867 \\ \mathrm{C} & 2.61 & -1.95098 & 0.38648 \\ \mathrm{C} & 2.65728 & -1.66941 & -1.04608 \\ \mathrm{H} & 3.16964 & -3.89226 & 1.05068 \\ \mathrm{C} & 3.18126 & -0.33452 & -1.20497 \\ \mathrm{C} & 4.04339 & 1.53431 & 0.43382 \\ \mathrm{H} & 3.55453 & 2.02967 & 1.27706 \\ \mathrm{H} & 4.03815 & 2.21834 & -0.41836 \\ \mathrm{H} & 5.09209 & 1.36386 & 0.70737 \\ \mathrm{C} & 3.2444 & -0.67215 & 2.56162 \\ \mathrm{H} & 4.28083 & -0.92932 & 2.81417 \\ \mathrm{H} & 2.58532 & -1.3447 & 3.11375 \\ \mathrm{H} & 3.04953 & 0.34351 & 2.91113 \\ \mathrm{H} & 1.26727 & -3.27034 & 0.99574 \\ \mathrm{H} & & -3.80376 & 0.4062\end{array}$

$\begin{array}{cccl}\mathrm{C} & 2.41983 & -2.66882 & -2.13161 \\ \mathrm{H} & 2.1991 & -2.1816 & -3.08432 \\ \mathrm{H} & 1.57838 & -3.32196 & -1.88906 \\ \mathrm{H} & 3.30923 & -3.2952 & -2.27429 \\ \mathrm{C} & 3.56217 & 0.29992 & -2.5025 \\ \mathrm{H} & 4.59234 & 0.0241 & -2.75702 \\ \mathrm{H} & 3.52138 & 1.39104 & -2.45302 \\ \mathrm{H} & 2.92191 & -0.03296 & -3.32316 \\ \mathrm{H} & -4.2399 & -1.71915 & -2.20237 \\ \mathrm{I} & -0.94302 & -1.94458 & -0.20518 \\ \mathrm{H} & -3.45053 & 3.45721 & -0.85056 \\ \mathrm{H} & -2.17533 & -0.00277 & -1.89433 \\ \mathrm{H} & -3.50615 & 1.10918 & -2.38127 \\ \mathrm{H} & 1.13368 & 1.31552 & -2.35412 \\ \mathrm{H} & -0.19191 & 0.1469 & -2.38024\end{array}$

\section{IrI $_{2} \mathbf{v y}: E=-1165.49663187$}

\begin{tabular}{|c|c|c|c|}
\hline Ir & 1.10433 & -0.09347 & -0.18713 \\
\hline $\mathrm{N}$ & -0.6255 & 3.78162 & -0.24544 \\
\hline $\mathrm{C}$ & -0.04105 & 1.5899 & -0.37014 \\
\hline I & 0.55325 & 0.1412 & 2.50876 \\
\hline $\mathrm{N}$ & -1.33891 & 1.84755 & -0.88018 \\
\hline $\mathrm{C}$ & 0.36264 & 2.85015 & 0.0175 \\
\hline $\mathrm{H}$ & 1.26489 & 3.14479 & 0.52741 \\
\hline N & -3.13492 & 0.35919 & -0.32516 \\
\hline $\mathrm{C}$ & -0.51278 & 5.2095 & 0.02726 \\
\hline $\mathrm{H}$ & -0.43861 & 5.77618 & -0.90387 \\
\hline $\mathrm{H}$ & 0.38938 & 5.3721 & 0.61577 \\
\hline $\mathrm{H}$ & -1.3701 & 5.55753 & 0.60644 \\
\hline $\mathrm{C}$ & -1.67093 & 3.165 & -0.79586 \\
\hline $\mathrm{N}$ & -4.56961 & -0.93468 & 0.64875 \\
\hline $\mathrm{C}$ & -2.93151 & 3.79209 & -1.26821 \\
\hline $\mathrm{H}$ & -3.8103 & 3.27474 & -0.87071 \\
\hline $\mathrm{H}$ & -2.99993 & 3.78987 & -2.36292 \\
\hline $\mathrm{H}$ & -2.98795 & 4.83137 & -0.94139 \\
\hline $\mathrm{C}$ & -2.24164 & 0.8587 & -1.38453 \\
\hline $\mathrm{H}$ & -1.65097 & 0.00541 & -1.7397 \\
\hline $\mathrm{H}$ & -2.85371 & 1.27507 & -2.18777 \\
\hline $\mathrm{C}$ & -2.94426 & 0.50564 & 1.03272 \\
\hline $\mathrm{H}$ & -2.13937 & 1.08959 & 1.45437 \\
\hline $\mathrm{C}$ & -3.85719 & -0.29439 & 1.64072 \\
\hline $\mathrm{H}$ & -4.03436 & -0.48136 & 2.68751 \\
\hline $\mathrm{C}$ & -5.61623 & -1.92633 & 0.87889 \\
\hline $\mathrm{H}$ & -6.54008 & -1.63083 & 0.37794 \\
\hline $\mathrm{H}$ & -5.80143 & -1.98268 & 1.95066 \\
\hline $\mathrm{H}$ & -5.28998 & -2.90615 & 0.52462 \\
\hline $\mathrm{C}$ & -4.10463 & -0.5475 & -0.54911 \\
\hline $\mathrm{C}$ & -4.53918 & -1.04103 & -1.87781 \\
\hline $\mathrm{H}$ & -4.79445 & -0.21421 & -2.54817 \\
\hline $\mathrm{H}$ & -5.41669 & -1.68175 & -1.7875 \\
\hline $\mathrm{C}$ & 3.15662 & 0.60218 & -0.23668 \\
\hline $\mathrm{C}$ & 3.22619 & -0.78504 & 0.21091 \\
\hline $\mathrm{C}$ & 2.68735 & -1.61098 & -0.80364 \\
\hline $\mathrm{C}$ & 2.26861 & -0.75892 & -1.91651 \\
\hline $\mathrm{C}$ & 2.6221 & 0.59501 & -1.58377 \\
\hline $\mathrm{C}$ & 3.80021 & 1.75069 & 0.47226 \\
\hline $\mathrm{H}$ & 3.53736 & 1.7553 & 1.5336 \\
\hline $\mathrm{H}$ & 3.5089 & 2.71005 & 0.03764 \\
\hline $\mathrm{H}$ & 4.89227 & 1.68058 & 0.39611 \\
\hline $\mathrm{C}$ & 3.84591 & -1.24046 & 1.49003 \\
\hline $\mathrm{H}$ & 4.93241 & -1.3222 & 1.35934 \\
\hline $\mathrm{H}$ & 3.46775 & -2.21744 & 1.79576 \\
\hline $\mathrm{H}$ & 3.64961 & -0.539 & 2.30334 \\
\hline $\mathrm{C}$ & 2.65733 & -3.10442 & -0.7 \\
\hline
\end{tabular}




$\begin{array}{llll}\mathrm{H} & 2.4767 & -3.49888 & 0.20593 \\ \mathrm{H} & 3.62247 & -3.49122 & -1.14644 \\ \mathrm{H} & 1.87574 & -3.49571 & -1.44888 \\ \mathrm{C} & 1.78504 & -1.25832 & -3.23902 \\ \mathrm{H} & 1.30574 & -0.46476 & -3.81698 \\ \mathrm{H} & 1.06139 & -2.06714 & -3.11178 \\ \mathrm{H} & 2.62523 & -1.64249 & -3.83067 \\ \mathrm{C} & 2.56127 & 1.76859 & -2.5061 \\ \mathrm{H} & 3.52327 & 1.88263 & -3.01972 \\ \mathrm{H} & 2.35814 & 2.69776 & -1.96903 \\ \mathrm{H} & 1.78939 & 1.6425 & -3.26871 \\ \mathrm{H} & -3.73457 & -1.62754 & -2.33648 \\ \mathrm{I} & -0.79573 & -2.14349 & -0.42799\end{array}$

IrIme : $\mathrm{E}=-1153.84364807$

\begin{tabular}{|c|c|c|c|}
\hline & -0.42596 & & \\
\hline & -2.69506 & 0.19257 & \\
\hline & -1.8843 & -0.30425 & 9313 \\
\hline & 0.8827 & & \\
\hline & -1.19776 & -1.00951 & \\
\hline & -2.81309 & & \\
\hline & -3.55242 & & \\
\hline & 1.14262 & -1.39276 & \\
\hline & -3.52549 & 0.79481 & \\
\hline & -2.88523 & 1.10245 & -0.0 \\
\hline & -4.02378 & 1.6 & \\
\hline & -4.27656 & & \\
\hline & -1.710 & -0 & 1.581 \\
\hline & 3.23331 & $-0 . \varepsilon$ & \\
\hline & -1.35 & -1.2 & \\
\hline & -0.16 & -1 & \\
\hline & -0.0 & -2 . & \\
\hline & -0.4 & -2 & \\
\hline & 1.4 & -0.6 & \\
\hline & 0.7 & -0 & \\
\hline & 2.7 & -0 . & \\
\hline & 3.3 & & \\
\hline & 4.6 & -0 . & \\
\hline & 5.0 & -1 & \\
\hline & 5.1 & -0 . & \\
\hline & 4.6 & -0 . & \\
\hline & 2.2 & -1 & \\
\hline & 2 & -2 & \\
\hline & 0.0 & 1 & -3 \\
\hline & 0.7 & & -3 \\
\hline & -0.241 & -1 & -3 \\
\hline & -1.54959 & -0 . & -2 \\
\hline & -1.36714 & & -2 \\
\hline & 0.6 & 2 . & -3 \\
\hline & 1.6 & 2. & -2 . \\
\hline & 0.03366 & 3.3 & -2 . \\
\hline & 0.7 & & -4 . \\
\hline & 2.2 & -0 . & -3 \\
\hline & 2.4 & -0 . & -4 \\
\hline & & -1 & -2 . \\
\hline & 2.76784 & & -2.7801 \\
\hline & 0.0 & -2 & -3 \\
\hline & 1.03836 & -2.7 & -2 . \\
\hline & 0.15642 & -2.7 & -4.23 \\
\hline & -0.69208 & -3.1 & -2.7339 \\
\hline & -2.85296 & -1.2 & -3.0812 \\
\hline & & -0.7 & -2.5301 \\
\hline & -2.78242 & -2.25469 & -2.713 \\
\hline & -3.16534 & -1.27917 & -4.130 \\
\hline
\end{tabular}

$\begin{array}{llll}\mathrm{C} & -2.45696 & 1.94905 & -2.9008 \\ \mathrm{H} & -2.62403 & 2.3 & -3.92739 \\ \mathrm{H} & -2.19447 & 2.82125 & -2.29785 \\ \mathrm{H} & -3.40219 & 1.53788 & -2.54214 \\ \mathrm{H} & -0.66334 & -2.07409 & 0.38576 \\ \mathrm{H} & -2.26582 & -1.63091 & -0.19656 \\ \mathrm{H} & -1.67842 & -0.41972 & 4.8451 \\ \mathrm{H} & 2.0098 & -3.3192 & 1.47995 \\ \mathrm{H} & 1.8294 & -1.79359 & 0.56038 \\ \mathrm{H} & 3.42565 & -2.36025 & 1.06429\end{array}$

IrIvy : E = -1154.20454789

\begin{tabular}{|c|c|c|c|}
\hline Ir & 1.29304 & -0.00569 & 0.07274 \\
\hline I & 0.03401 & -2.09895 & -0.80663 \\
\hline $\mathrm{N}$ & -1.55697 & 1.37172 & 0.22808 \\
\hline C & 2.85173 & 0.85818 & 1.39169 \\
\hline C & 3.16938 & 1.27489 & 0.0603 \\
\hline C & 3.53312 & 0.07702 & -0.70464 \\
\hline $\mathrm{C}$ & 2.65707 & 1.73905 & 2.57698 \\
\hline $\mathrm{H}$ & 2.02613 & 1.27668 & 3.33863 \\
\hline $\mathrm{H}$ & 2.22885 & 2.70556 & 2.30693 \\
\hline $\mathrm{H}$ & 3.63517 & 1.92887 & 3.039 \\
\hline C & 3.40353 & 2.67683 & -0.39142 \\
\hline $\mathrm{H}$ & 3.22427 & 2.81162 & -1.46063 \\
\hline $\mathrm{H}$ & 4.45722 & 2.92999 & -0.21422 \\
\hline $\mathrm{H}$ & 2.80893 & 3.39703 & 0.17394 \\
\hline C & 4.00634 & 0.06878 & -2.10652 \\
\hline $\mathrm{H}$ & 5.104 & 0.17185 & -2.09165 \\
\hline $\mathrm{H}$ & 3.62204 & 0.90621 & -2.69256 \\
\hline $\mathrm{H}$ & 3.78881 & -0.87072 & -2.61896 \\
\hline C & -0.18032 & 1.36926 & -0.12243 \\
\hline C & -2.14099 & 2.53474 & -0.15823 \\
\hline C & -2.25561 & 0.34214 & 0.98804 \\
\hline $\mathrm{H}$ & -2.57171 & 0.7389 & 1.95522 \\
\hline $\mathrm{H}$ & -1.54695 & -0.47088 & 1.14955 \\
\hline $\mathrm{N}$ & -3.43602 & -0.17612 & 0.29729 \\
\hline $\mathrm{C}$ & 2.97692 & -0.61115 & 1.4339 \\
\hline $\mathrm{C}$ & 3.39334 & -1.07451 & 0.13695 \\
\hline C & 2.87288 & -1.44692 & 2.65662 \\
\hline $\mathrm{H}$ & 2.55867 & -2.46863 & 2.43779 \\
\hline $\mathrm{H}$ & 3.87215 & -1.50397 & 3.11301 \\
\hline $\mathrm{H}$ & 2.20597 & -1.01633 & 3.40622 \\
\hline $\mathrm{C}$ & 3.75303 & -2.47477 & -0.22679 \\
\hline $\mathrm{H}$ & 4.83131 & -2.61436 & -0.07749 \\
\hline $\mathrm{H}$ & 3.23695 & -3.20711 & 0.39552 \\
\hline $\mathrm{H}$ & 3.53305 & -2.69951 & -1.27233 \\
\hline $\mathrm{C}$ & -3.50356 & -0.50694 & -1.04763 \\
\hline $\mathrm{C}$ & -4.59318 & -0.54609 & 0.9069 \\
\hline $\mathrm{N}$ & -1.20907 & 3.26336 & -0.78538 \\
\hline $\mathrm{N}$ & -5.37663 & -1.08743 & -0.03678 \\
\hline $\mathrm{C}$ & -0.01539 & 2.58491 & -0.75254 \\
\hline C & -4.72313 & -1.06643 & -1.2517 \\
\hline $\mathrm{H}$ & 0.86722 & 3.01383 & -1.19871 \\
\hline $\mathrm{H}$ & -5.17651 & -1.45075 & -2.15338 \\
\hline $\mathrm{C}$ & -6.72924 & -1.62854 & 0.17398 \\
\hline $\mathrm{H}$ & -6.70705 & -2.39693 & 0.94797 \\
\hline $\mathrm{H}$ & -7.4169 & -0.82679 & 0.44965 \\
\hline $\mathrm{H}$ & -7.06457 & -2.07799 & -0.75969 \\
\hline C & -1.41644 & 4.58703 & -1.39188 \\
\hline $\mathrm{H}$ & -2.23265 & 4.54462 & -2.11444 \\
\hline $\mathrm{H}$ & -1.63266 & 5.32557 & -0.6168 \\
\hline $\mathrm{H}$ & -0.50225 & 4.87311 & -1.91025 \\
\hline $\mathrm{C}$ & -4.93059 & -0.43046 & 2.34804 \\
\hline $\mathrm{H}$ & -4.48816 & -1.25062 & 2.92622 \\
\hline
\end{tabular}




$\begin{array}{lrrr}\mathrm{H} & -4.58808 & 0.51435 & 2.77848 \\ \mathrm{H} & -6.01193 & -0.47353 & 2.48918 \\ \mathrm{C} & -3.54695 & 2.95223 & 0.05877 \\ \mathrm{H} & -4.20623 & 2.55616 & -0.72252 \\ \mathrm{H} & -3.91877 & 2.61495 & 1.02897 \\ \mathrm{H} & -3.62746 & 4.04085 & 0.04001 \\ \mathrm{H} & 0.57529 & -0.29176 & 1.41819 \\ \mathrm{H} & -2.692 & -0.32431 & -1.73302\end{array}$

TS2meme : $\mathrm{E}=-1153.80443245$

\begin{tabular}{|c|c|c|c|}
\hline $\operatorname{Ir}$ & 0.89397 & -0.12236 & -0.25915 \\
\hline $\mathrm{N}$ & -1.81232 & 2.93223 & -0.24811 \\
\hline $\mathrm{C}$ & -3.76813 & 2.02483 & -0.70331 \\
\hline$I$ & -0.77859 & 0.21114 & 1.88899 \\
\hline $\mathrm{N}$ & -2.74834 & 1.27245 & -1.27025 \\
\hline $\mathrm{C}$ & -3.17602 & 3.06048 & -0.06583 \\
\hline $\mathrm{H}$ & -3.60515 & 3.88042 & 0.48881 \\
\hline $\mathrm{N}$ & -2.95201 & -1.12735 & -1.15736 \\
\hline $\mathrm{C}$ & -0.81432 & 3.84359 & 0.30057 \\
\hline $\mathrm{H}$ & -0.16145 & 3.29574 & 0.98 \\
\hline $\mathrm{H}$ & -1.33061 & 4.6274 & 0.85 \\
\hline $\mathrm{H}$ & -0.23867 & 4.3038 & -0.50574 \\
\hline $\mathrm{C}$ & -1.54386 & 1.83168 & -0.97 \\
\hline $\mathrm{N}$ & -2.31069 & -2.81495 & 0.03 \\
\hline $\mathrm{C}$ & -0.22585 & 1.36203 & -1.39 \\
\hline $\mathrm{C}$ & -2.94855 & 0.04987 & -2.01 \\
\hline $\mathrm{H}$ & -2.17295 & -0.05425 & -2.77 \\
\hline $\mathrm{H}$ & -3.91229 & 0.10249 & -2.52 \\
\hline $\mathrm{C}$ & -4.07192 & -1.61027 & -0.5 \\
\hline $\mathrm{H}$ & -5.0533 & -1.18366 & -0.6 \\
\hline $\mathrm{C}$ & -3.66629 & -2.6658 & 0.2 \\
\hline $\mathrm{H}$ & -4.22557 & -3.32597 & 0.8 \\
\hline $\mathrm{C}$ & -1.47609 & -3.81362 & 0.7 \\
\hline $\mathrm{H}$ & -0.91706 & -4.39321 & -0.0 \\
\hline $\mathrm{H}$ & -2.12516 & -4.49088 & 1.25 \\
\hline $\mathrm{H}$ & -0.8005 & -3.31753 & 1.4 \\
\hline $\mathrm{C}$ & -1.87228 & -1.86919 & -0.81 \\
\hline $\mathrm{C}$ & -0.48836 & -1.70419 & -1.27 \\
\hline $\mathrm{C}$ & 2.71545 & -0.53849 & 0.95398 \\
\hline $\mathrm{C}$ & 2.78734 & -1.29709 & -0.25943 \\
\hline $\mathrm{C}$ & 2.8053 & -0.37444 & -1.38 \\
\hline $\mathrm{C}$ & 2.80999 & 0.95908 & -0.84 \\
\hline $\mathrm{C}$ & 2.69027 & 0.87337 & 0.58951 \\
\hline $\mathrm{C}$ & 2.82127 & -1.06306 & 2.34748 \\
\hline $\mathrm{H}$ & 2.46949 & -2.0933 & 2.4268 \\
\hline $\mathrm{H}$ & 2.25016 & -0.45655 & 3.0 \\
\hline $\mathrm{H}$ & 3.87209 & -1.04528 & 2.66 \\
\hline $\mathrm{C}$ & 2.95425 & -2.77805 & -0.36 \\
\hline $\mathrm{H}$ & 4.02246 & -3.01343 & -0.4 \\
\hline $\mathrm{H}$ & 2.47665 & -3.18527 & $-1 \cdot 2$ \\
\hline $\mathrm{H}$ & 2.56726 & -3.30076 & 0.50 \\
\hline $\mathrm{C}$ & 3.02859 & -0.74305 & -2.81544 \\
\hline $\mathrm{H}$ & 2.58454 & -1.70957 & -3.06387 \\
\hline $\mathrm{H}$ & 4.10409 & -0.81944 & -3.01654 \\
\hline $\mathrm{H}$ & 2.62277 & 0.00587 & -3.49903 \\
\hline $\mathrm{C}$ & 2.99645 & 2.20904 & -1.63341 \\
\hline $\mathrm{H}$ & 2.59854 & 3.08925 & -1.12431 \\
\hline $\mathrm{H}$ & 2.55607 & 2.14451 & -2.63113 \\
\hline $\mathrm{H}$ & 4.07227 & 2.37843 & -1.76662 \\
\hline $\mathrm{C}$ & 2.77644 & 2.00217 & 1.56267 \\
\hline $\mathrm{H}$ & 3.81194 & 2.10688 & 1.90846 \\
\hline $\mathrm{H}$ & 2.14835 & 1.82293 & 2.4378 \\
\hline $\mathrm{H}$ & 2.48478 & 2.95406 & 1.11 \\
\hline $\mathrm{H}$ & -0.29744 & 0.97248 & -2.4 \\
\hline
\end{tabular}

$\begin{array}{rrrr}\mathrm{H} & 0.42828 & 2.23406 & -1.45197 \\ \mathrm{H} & -4.81096 & 1.77697 & -0.82375 \\ \mathrm{H} & 0.24767 & -1.55953 & 0.02059 \\ \mathrm{H} & -0.02693 & -2.68772 & -1.40425 \\ \mathrm{H} & -0.44785 & -1.21754 & -2.24823\end{array}$

TS2vyme : $\mathrm{E}=-1153.81279463$

\begin{tabular}{|c|c|c|c|}
\hline Ir & 0.04132 & -0.28988 & -0.79576 \\
\hline $\mathrm{N}$ & -3.07645 & -0.10721 & 2.14898 \\
\hline C & -1.73423 & -0.74429 & 3.77819 \\
\hline I & -0.73089 & 2.12702 & 0.24259 \\
\hline $\mathrm{N}$ & -1.24816 & -1.19251 & 2.5628 \\
\hline C & -2.87969 & -0.06744 & .51454 \\
\hline $\mathrm{H}$ & -3.5716 & 0.42716 & 4.1786 \\
\hline $\mathrm{N}$ & 1.10718 & -1.05712 & .054 \\
\hline C & -4.2022 & 0.51624 & .44 \\
\hline $\mathrm{H}$ & -3.82548 & 1.29106 & $0.7^{\circ}$ \\
\hline $\mathrm{H}$ & -4.85516 & 0.971 & 2.1 \\
\hline $\mathrm{H}$ & -4.76582 & -0.23789 & 0.8 \\
\hline C & -2.07 & -0.78238 & 1.5715 \\
\hline $\mathrm{N}$ & 2.92 & 0.06 & 2.3 \\
\hline C & -1.917 & -1.086 & 0.1 \\
\hline C & -0.00 & -1.926 & 2.3 \\
\hline $\mathrm{H}$ & -0.14 & -2.64 & 1 \\
\hline $\mathrm{H}$ & 0.20 & -2.48238 & 3.2 \\
\hline $\mathrm{C}$ & 1.26434 & -0.33892 & 0.8 \\
\hline $\mathrm{H}$ & -0.56 & -1.62639 & -0.1 \\
\hline $\mathrm{C}$ & 2.42 & 0.35 & 1.0 \\
\hline $\mathrm{H}$ & 2.90 & 1.091 & 0.4 \\
\hline $\mathrm{C}$ & 4.16 & 0.63 & 2.8 \\
\hline $\mathrm{H}$ & 3.95 & 1.12 & 3.8 \\
\hline $\mathrm{H}$ & 4.53 & 1.38 & 2.1 \\
\hline $\mathrm{H}$ & 4.91 & -0.14 & 2.9 \\
\hline $\mathrm{C}$ & 2.12 & -0.79 & 2.9 \\
\hline $\mathrm{C}$ & 2.31 & -1.38828 & 4.2 \\
\hline $\mathrm{H}$ & 1.52 & -1.10373 & 4.9 \\
\hline $\mathrm{H}$ & 3.25 & -1.03902 & 4.7 \\
\hline $\mathrm{C}$ & -0.65 & -0.58412 & -2.9 \\
\hline C & 0.01 & 0.66784 & $-2 . \varepsilon$ \\
\hline C & 1.36 & 0.44343 & -2 \\
\hline $\mathrm{C}$ & 1.55 & -0.98608 & -2.2 \\
\hline $\mathrm{C}$ & 0.29 & -1.61598 & -2.5 \\
\hline $\mathrm{C}$ & -2.029 & -0.82367 & -3.4 \\
\hline $\mathrm{H}$ & -2.68613 & 0.03111 & -3.315 \\
\hline $\mathrm{H}$ & -2.49072 & -1.71218 & -3.0 \\
\hline $\mathrm{H}$ & -1.99126 & -0.98397 & -4.5784 \\
\hline C & -0.516 & 1.97726 & -3.37078 \\
\hline $\mathrm{H}$ & -0.25493 & 2.0911 & -4.4 \\
\hline $\mathrm{H}$ & -0.08294 & 2.81653 & -2.825 \\
\hline $\mathrm{H}$ & -1.60217 & 2.03978 & -3.28 \\
\hline C & 2.438 & 1.48724 & -2.35709 \\
\hline $\mathrm{H}$ & 2.102 & 2.3966 & -1.85224 \\
\hline $\mathrm{H}$ & 2.718 & 1.76078 & -3.381 \\
\hline $\mathrm{H}$ & 3.34258 & 1.12328 & -1.86642 \\
\hline $\mathrm{C}$ & 2.84339 & -1.68377 & -1.98243 \\
\hline $\mathrm{H}$ & 2.68962 & -2.67031 & -1.54138 \\
\hline $\mathrm{H}$ & 3.48474 & -1.10684 & -1.31391 \\
\hline $\mathrm{H}$ & 3.38577 & -1.82172 & -2.92538 \\
\hline $\mathrm{C}$ & 0.05155 & -3.08775 & -2.661 \\
\hline $\mathrm{H}$ & 0.25491 & -3.41622 & -3.68777 \\
\hline $\mathrm{H}$ & -0.98523 & -3.35212 & -2.4408 \\
\hline $\mathrm{H}$ & 0.70528 & -3.65854 & -1.999 \\
\hline $\mathrm{H}$ & 2.37241 & -2.48149 & 4.2 \\
\hline$H$ & & -0 & \\
\hline
\end{tabular}


$\mathrm{H} \quad-2.55755-0.4396 \quad-0.46173$

$\mathrm{H} \quad-2.24273-2.11761 \quad-0.06253$

IrImemeH $^{+}: \mathrm{E}=-1153.82084167$

\begin{tabular}{|c|c|c|c|}
\hline Ir & -0.84325 & 0.16693 & -0.21082 \\
\hline $\mathrm{N}$ & 2.05063 & -2.79169 & -0.33096 \\
\hline C & 3.93355 & -1.73866 & -0.77845 \\
\hline I & 0.80914 & -0.32526 & 1.93061 \\
\hline $\mathrm{N}$ & 2.85696 & -1.02523 & -1.28134 \\
\hline $\mathrm{C}$ & 3.42304 & -2.84354 & -0.18561 \\
\hline $\mathrm{H}$ & 3.91628 & -3.66307 & 0.31364 \\
\hline $\mathrm{N}$ & 2.78715 & 1.37831 & -1.08905 \\
\hline C & 1.12746 & -3.80386 & 17075 \\
\hline $\mathrm{H}$ & 0.41843 & -3.33761 & 0.85726 \\
\hline $\mathrm{H}$ & 1.70192 & -4.5555 & 0.71067 \\
\hline $\mathrm{H}$ & 0.60818 & -4.28771 & -0.65976 \\
\hline C & 1.69855 & -1.67143 & -0.98828 \\
\hline N & 1.88905 & 2.96257 & 0.07771 \\
\hline C & 0.34533 & -1.27467 & -1.38251 \\
\hline $\mathrm{C}$ & 2.95972 & 0.24239 & -1.97264 \\
\hline $\mathrm{H}$ & 2.21718 & 0.28733 & -2.76 \\
\hline $\mathrm{H}$ & 3.94842 & 0.30584 & -2.42923 \\
\hline C & 3.81025 & 1.98258 & -0.37633 \\
\hline $\mathrm{H}$ & 4.84101 & 1.67729 & -0.46 \\
\hline C & 3.24358 & 2.97254 & 0.35225 \\
\hline $\mathrm{H}$ & 3.68559 & 3.68921 & 1.02708 \\
\hline C & 0.92337 & 3.87146 & 0.68654 \\
\hline $\mathrm{H}$ & 0.2675 & 4.29193 & -0.0771 \\
\hline $\mathrm{H}$ & 1.46832 & 4.68622 & 1.16183 \\
\hline $\mathrm{H}$ & 0.34016 & 3.34282 & 1.44496 \\
\hline C & 1.60561 & 1.97942 & -0.79713 \\
\hline $\mathrm{C}$ & 0.28519 & 1.62057 & -1.32972 \\
\hline $\mathrm{C}$ & -2.81235 & 0.47979 & 0.8019 \\
\hline $\mathrm{C}$ & -2.81021 & 1.07465 & -0.5229 \\
\hline $\mathrm{C}$ & -2.65005 & 0.00517 & -1.48226 \\
\hline $\mathrm{C}$ & -2.64743 & -1.24634 & -0.74025 \\
\hline $\mathrm{C}$ & -2.72213 & -0.95609 & 0.65295 \\
\hline $\mathrm{C}$ & -3.08648 & 1.17837 & 2.09384 \\
\hline $\mathrm{H}$ & -2.90525 & 2.25219 & 2.02751 \\
\hline $\mathrm{H}$ & -2.4772 & 0.77624 & 2.90625 \\
\hline $\mathrm{H}$ & -4.13935 & 1.03528 & 2.36345 \\
\hline C & -3.11685 & 2.50023 & -0.84694 \\
\hline $\mathrm{H}$ & -4.20025 & 2.61386 & -0.97181 \\
\hline $\mathrm{H}$ & -2.65188 & 2.82095 & -1.78177 \\
\hline $\mathrm{H}$ & -2.80735 & 3.17953 & -0.05017 \\
\hline C & -2.74536 & 0.13941 & -2.96828 \\
\hline $\mathrm{H}$ & -2.37454 & 1.10732 & -3.31218 \\
\hline $\mathrm{H}$ & -3.7924 & 0.05987 & -3.28402 \\
\hline $\mathrm{H}$ & -2.18923 & -0.64387 & -3.48854 \\
\hline C & -2.70046 & -2.60324 & -1.35296 \\
\hline $\mathrm{H}$ & -2.27715 & -3.37198 & -0.70286 \\
\hline $\mathrm{H}$ & -2.21024 & -2.64517 & -2.32838 \\
\hline $\mathrm{H}$ & -3.75255 & -2.86878 & -1.51803 \\
\hline C & -2.82626 & -1.93716 & 1.77173 \\
\hline $\mathrm{H}$ & -3.87944 & -2.0576 & 2.05402 \\
\hline $\mathrm{H}$ & -2.28277 & -1.59883 & 2.65665 \\
\hline $\mathrm{H}$ & -2.44795 & -2.92189 & 1.4928 \\
\hline $\mathrm{H}$ & 0.37159 & -0.88586 & -2.40519 \\
\hline $\mathrm{H}$ & -0.25628 & -2.1834 & -1.40471 \\
\hline $\mathrm{H}$ & 4.95521 & -1.41637 & -0.90454 \\
\hline $\mathrm{H}$ & -0.63142 & 1.42067 & 0.71259 \\
\hline $\mathrm{H}$ & -0.32311 & 2.52606 & -1.38058 \\
\hline $\mathrm{H}$ & 0.38935 & 1.2532 & -2.35321 \\
\hline
\end{tabular}

IrIvymeH $^{+}$: E = -1153.83007053

\begin{tabular}{|c|c|c|c|}
\hline Ir & -0.74141 & 0.12026 & -0.39204 \\
\hline $\mathrm{N}$ & 2.02507 & -3.09914 & -0.37311 \\
\hline C & 3.73425 & -1.79823 & -0.86946 \\
\hline I & 0.16831 & -0.91548 & 2.00562 \\
\hline $\mathrm{N}$ & 2.54785 & -1.18874 & -1.24165 \\
\hline C & 3.4018 & -2.99494 & -0.32767 \\
\hline $\mathrm{H}$ & 4.02305 & -3.78107 & 0.07269 \\
\hline $\mathrm{N}$ & 2.14592 & 1.16223 & -0.872 \\
\hline $\mathrm{C}$ & 1.26641 & -4.25284 & \\
\hline $\mathrm{H}$ & 0.58385 & -3.93507 & 0.9016 \\
\hline $\mathrm{H}$ & 1.97038 & -4.97772 & \\
\hline $\mathrm{H}$ & 0.71709 & -4.71516 & -0.71141 \\
\hline C & 1.50208 & -1.98826 & -0.92 \\
\hline $\mathrm{N}$ & 2.42977 & 2.85616 & \\
\hline $\mathrm{C}$ & 0.07884 & -1.70268 & -1.177 \\
\hline $\mathrm{C}$ & 2.42538 & 0.12504 & -1.84 \\
\hline $\mathrm{H}$ & 1.62 & 0.10063 & -2.5 \\
\hline $\mathrm{H}$ & 3.35799 & 0.35102 & $-2 \cdot 3$ \\
\hline $\mathrm{C}$ & 0.930 & 1.30052 & -0.1 \\
\hline $\mathrm{H}$ & -0.3084 & 0.46866 & -1.8 \\
\hline $\mathrm{C}$ & 1.14 & 2.38096 & 0.62 \\
\hline $\mathrm{H}$ & 0.50283 & 2.82903 & 1.3 \\
\hline C & 3.00771 & 4.00593 & 1.13338 \\
\hline $\mathrm{H}$ & 3.92409 & 3.71457 & 1.64 \\
\hline $\mathrm{H}$ & 2.28 & 4.35098 & 1.8734 \\
\hline $\mathrm{H}$ & 3.21054 & 4.81702 & 0.4 \\
\hline C & 3.04 & 2.10713 & -0.4 \\
\hline C & 4.42 & 2.29973 & -1.0 \\
\hline $\mathrm{H}$ & 5.06396 & 1.44243 & -0.77789 \\
\hline $\mathrm{H}$ & 4.88005 & 3.17485 & -0.5 \\
\hline $\mathrm{C}$ & -2.87505 & -0.62574 & -0.05857 \\
\hline C & -2.7413 & 0.47201 & 0.89168 \\
\hline $\mathrm{C}$ & -2.41689 & 1.66397 & 0.1757 \\
\hline C & -2.39514 & 1.33864 & -1.22681 \\
\hline $\mathrm{C}$ & -2.7169 & -0.08612 & -1.37089 \\
\hline C & -3.306 & -2.01036 & 0.29967 \\
\hline $\mathrm{H}$ & -2.77541 & -2.37462 & 1.1 \\
\hline $\mathrm{H}$ & -3.14044 & -2.71301 & -0.51 \\
\hline $\mathrm{H}$ & -4.37844 & -2.02206 & 0.52 \\
\hline C & -3.05605 & 0.39343 & 2.3 \\
\hline $\mathrm{H}$ & -4.11568 & 0.65756 & 2.4 \\
\hline $\mathrm{H}$ & -2.46756 & 1.0957 & 2.9 \\
\hline $\mathrm{H}$ & -2.91148 & -0.60887 & 2.74466 \\
\hline $\mathrm{C}$ & -2.32232 & 3.03814 & 0.75 \\
\hline $\mathrm{H}$ & -1.96775 & 3.02119 & 1.7 \\
\hline $\mathrm{H}$ & -3.31524 & 3.50378 & 0.7 \\
\hline $\mathrm{H}$ & -1.66624 & 3.68351 & 0.16661 \\
\hline $\mathrm{C}$ & -2.33278 & 2.33577 & -2.33777 \\
\hline $\mathrm{H}$ & -2.04253 & 1.88034 & -3.28607 \\
\hline $\mathrm{H}$ & -1.63936 & 3.14956 & -2.11698 \\
\hline $\mathrm{H}$ & -3.32749 & 2.77739 & -2.4739 \\
\hline $\mathrm{C}$ & -2.99585 & -0.7889 & -2.65952 \\
\hline $\mathrm{H}$ & -4.05815 & -0.68043 & -2.90794 \\
\hline $\mathrm{H}$ & -2.7851 & -1.85851 & -2.59683 \\
\hline $\mathrm{H}$ & -2.42122 & -0.37255 & -3.48964 \\
\hline $\mathrm{H}$ & 4.42665 & 2.4657 & -2.08497 \\
\hline $\mathrm{H}$ & 4.6998 & -1.35015 & -1.04285 \\
\hline $\mathrm{H}$ & -0.51542 & -2.50778 & -0.74401 \\
\hline $\mathrm{H}$ & -0.08 & -1.71936 & -2.2 \\
\hline
\end{tabular}

IrImeme : $\mathrm{E}=-1153.40590513$

$\begin{array}{crrr}\text { Ir } & 0.16968 & 0.32415 & 0.84551 \\ \text { N } & -3.18889 & -0.65239 & -1.00727 \\ \text { C } & -2.91646 & 0.00536 & -3.09186 \\ \text { I } & 0.1942 & -1.7908 & -0.97596\end{array}$




\begin{tabular}{|c|c|c|c|}
\hline $\mathrm{N}$ & -2.22485 & 0.84803 & -2.231 \\
\hline C & -3.51647 & -0.929 & -2.32181 \\
\hline & -4.14778 & -1.76097 & 58952 \\
\hline & 0.00085 & 1.46599 & 91477 \\
\hline & -3.55316 & -1.46682 & 059 \\
\hline & -2.64021 & -1.8438 & 0905 \\
\hline & -4.1543 & -2.30627 & 20697 \\
\hline & -4.1376 & -0.88088 & 42 \\
\hline & -2.38298 & 0.42627 & 9466 \\
\hline & 2.03629 & 0.79953 & -2 \\
\hline & -1.7978 & 1.00449 & \\
\hline & -1.34 & 1.91672 & -2 \\
\hline & $-1 \cdot 32$ & 2.68 & -1 \\
\hline & -1.75 & 2.35 & -3 \\
\hline & 0.43 & 0.93 & -4 \\
\hline & -0.18 & 0.91 & -5 \\
\hline & 1.70 & 0.52 & -3 \\
\hline & 2.41 & 0.06 & -4 \\
\hline & 3.28 & 0.43 & -1 \\
\hline & 3.78 & 1.32 & -1 \\
\hline & 3.927 & -0.05 & -2. \\
\hline & 3.06213 & -0.26 & -1 \\
\hline & 0.98664 & 1.36 & -1.9 \\
\hline & 0.92619 & 1.7 & -0 \\
\hline & 0.31 & 2.6 & -0 \\
\hline & 1.9 & 2.0 & -0 . \\
\hline 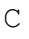 & 1.62 & -0.6 & \\
\hline$C$ & $1.7 \varepsilon$ & 0.7 & 2 . \\
\hline $\mathrm{C}$ & 0.5 & 1.3 & 2. \\
\hline C & -0.35 & 0.22 & 451 \\
\hline C & 0.27156 & -0.9 & 2 . \\
\hline C & 2.6 & $-1 \cdot 67$ & 443 \\
\hline $\mathrm{H}$ & 3.54 & -1.22 & 1. \\
\hline $\mathrm{H}$ & 2.31022 & -2.48 & 1.3 \\
\hline $\mathrm{H}$ & 3.0679 & -2.10 & 2. \\
\hline C & 3.04886 & 1.51 & 2 \\
\hline $\mathrm{H}$ & 3.56305 & 1.6 & 3. \\
\hline $\mathrm{H}$ & 2.86509 & 2.522 & 1.7 \\
\hline $\mathrm{H}$ & 3.73695 & 1.00 & 1.4 \\
\hline C & 0.28092 & 2.76 & 3. \\
\hline $\mathrm{H}$ & 0.8545 & 3.44169 & 2.4 \\
\hline $\mathrm{H}$ & 0.58402 & 2.9 & 4.1 \\
\hline $\mathrm{H}$ & -0.77338 & 3.03522 & 3. \\
\hline C & -1.75037 & 0.345 & 3.5 \\
\hline $\mathrm{H}$ & -2.4015 & -0.4867 & 3.3 \\
\hline $\mathrm{H}$ & -2.24693 & 1.27924 & 3.3 \\
\hline $\mathrm{H}$ & -1.66201 & 0.35812 & 4.6 \\
\hline $\mathrm{C}$ & -0.2365 & -2.38614 & \\
\hline $\mathrm{H}$ & 0.19978 & -2.8186 & 3.7 \\
\hline $\mathrm{H}$ & 0.02672 & -3.0327 & 2.0 \\
\hline $\mathrm{H}$ & -1.32242 & -2.4079 & 2.97629 \\
\hline $\mathrm{H}$ & -1.74393 & 2.09346 & 0.14057 \\
\hline $\mathrm{H}$ & -2.46919 & 0.79834 & \\
\hline $\mathrm{H}$ & -2.93148 & 0.15464 & -4 \\
\hline
\end{tabular}

IrIvyme : $\mathrm{E}=-1153.41495356$

$\begin{array}{crcc}\text { Ir } & 0.71633 & -0.24156 & 0.29848 \\ \mathrm{~N} & -2.69068 & -2.07015 & -1.44781 \\ \mathrm{C} & -1.07878 & -0.99532 & -0.27933 \\ \mathrm{I} & 0.47839 & 1.46374 & -1.86926 \\ \mathrm{~N} & -2.32315 & -0.99319 & 0.3808 \\ \mathrm{C} & -1.35968 & -1.6911 & -1.43396 \\ \mathrm{H} & -0.71974 & -1.8948 & -2.27723 \\ \mathrm{~N} & -2.5367 & 1.17138 & 1.3548\end{array}$

$$
\begin{aligned}
& \begin{array}{lll}
-3.32247 & -2.83735 & -2.51434
\end{array} \\
& -3.55205-3.85176 \quad-2.1789 \\
& -2.62693-2.89013-3.3511 \\
& -4.23395-2.34255-2.8544 \\
& \begin{array}{lll}
-3.28295 & -1.63497 & -0.33233
\end{array} \\
& \begin{array}{lll}
-1.70935 & 3.10091 & 0.83299
\end{array} \\
& \begin{array}{lll}
-4.69982 & -1.8528 & 0.06107
\end{array} \\
& \begin{array}{lll}
-5.20054 & -2.49597 & -0.66387
\end{array} \\
& \begin{array}{lll}
-5.26453 & -0.91501 & 0.10645
\end{array} \\
& \begin{array}{lll}
-4.77687 & -2.34556 & 1.03623
\end{array} \\
& \begin{array}{lll}
-2.56472 & -0.25511 & 1.60028
\end{array} \\
& \begin{array}{lll}
-1.78104 & -0.50249 & 2.31624
\end{array} \\
& \begin{array}{lll}
-3.53448 & -0.52442 & 2.01934
\end{array} \\
& \begin{array}{lll}
-3.60075 & 1.96912 & 0.96144
\end{array} \\
& \begin{array}{lll}
-4.62155 & 1.62284 & 0.96989
\end{array} \\
& \begin{array}{lll}
-3.07868 & 3.17636 & 0.64178
\end{array} \\
& \begin{array}{lll}
-3.5576 & 4.08254 & 0.30679
\end{array} \\
& \begin{array}{lll}
-0.76966 & 4.19534 & 0.61326
\end{array} \\
& \begin{array}{lll}
-0.29277 & 4.47697 & 1.55499
\end{array} \\
& \begin{array}{lll}
-1.32361 & 5.04879 & 0.22351
\end{array} \\
& \begin{array}{llll}
-0.02173 & 3.88417 & -0.12018
\end{array} \\
& \begin{array}{lll}
-1.37446 & 1.86707 & 1.25585
\end{array} \\
& \begin{array}{lll}
-0.05935 & 1.33018 & 1.55934
\end{array} \\
& \begin{array}{lll}
-0.07833 & 0.92893 & 2.58099
\end{array} \\
& \begin{array}{lll}
0.66917 & 2.14231 & 1.53326
\end{array} \\
& 2.38776-1.51455-0.47351 \\
& 2.98091-0.33841 \quad 0.15811 \\
& 2.60184-0.3236 \quad 1.52444 \\
& 1.73248-1.46913 \quad 1.77072 \\
& \begin{array}{lll}
1.66398 & -2.22529 & 0.53451
\end{array} \\
& 2.67197-1.96004-1.87229 \\
& 2.61697-1.12279-2.5732 \\
& 1.96496-2.72424-2.20383 \\
& 3.67794-2.39219-1.94122 \\
& \begin{array}{lll}
3.91942 & 0.60178 & -0.52516
\end{array} \\
& \begin{array}{llll}
4.90599 & 0.13254 & -0.62342
\end{array} \\
& \begin{array}{lll}
4.04539 & 1.53039 & 0.03464
\end{array} \\
& \begin{array}{lll}
3.5636 & 0.86193 & -1.52487
\end{array} \\
& \begin{array}{lll}
3.06633 & 0.64572 & 2.56453
\end{array} \\
& 3.34303 \quad 1.60775 \quad 2.1272 \\
& \begin{array}{lll}
3.95006 & 0.25091 & 3.07955
\end{array} \\
& \begin{array}{lll}
2.30289 & 0.82766 & 3.32568
\end{array} \\
& \begin{array}{lll}
1.2688 & -1.93167 & 3.11666
\end{array} \\
& \begin{array}{lll}
0.39631 & -2.58563 & 3.03798
\end{array} \\
& \begin{array}{lll}
1.00378 & -1.09057 & 3.76401
\end{array} \\
& \begin{array}{lll}
2.05804 & -2.49633 & 3.6287
\end{array} \\
& \begin{array}{lll}
1.02799 & -3.56914 & 0.38132
\end{array} \\
& \begin{array}{lll}
1.75416 & -4.35157 & 0.63199
\end{array} \\
& 0.69089-3.74572 \quad-0.64211 \\
& 0.16865-3.68924 \quad 1.04531
\end{aligned}
$$

Irme_ene : $\mathrm{E}=-1153.85235709$

$\begin{array}{lrrr}\text { Ir } & -0.34826 & -0.34022 & 0.67298 \\ \mathrm{~N} & 3.38389 & 0.11468 & -1.1998 \\ \mathrm{C} & 2.89861 & -0.57786 & -3.23509 \\ \mathrm{I} & 0.28932 & 1.93343 & -0.70654 \\ \mathrm{~N} & 2.01603 & -1.13049 & -2.32044 \\ \mathrm{C} & 3.75245 & 0.19987 & -2.52649 \\ \mathrm{H} & 4.59201 & 0.7946 & -2.85161 \\ \mathrm{~N} & -0.36143 & -1.4522 & -2.22324 \\ \mathrm{C} & 4.03781 & 0.84233 & -0.11517 \\ \mathrm{H} & 3.32802 & 1.54311 & 0.32967 \\ \mathrm{H} & 4.87628 & 1.39816 & -0.53278 \\ \mathrm{H} & 4.41838 & 0.14464 & 0.63342\end{array}$




\begin{tabular}{|c|c|c|c|}
\hline $\mathrm{C}$ & 2.31406 & -0.69416 & -1.06926 \\
\hline $\mathrm{N}$ & -2.06439 & -0.10422 & -2.03142 \\
\hline $\mathrm{C}$ & 1.64237 & -1.07613 & 0.18008 \\
\hline C & 0.89976 & -1.9975 & -2.6635 \\
\hline $\mathrm{H}$ & 1.02278 & -2.97759 & -2.19461 \\
\hline $\mathrm{H}$ & 0.90266 & -2.13559 & -3.74538 \\
\hline C & -0.88347 & -1.70234 & -0.92104 \\
\hline $\mathrm{H}$ & -0.8742 & -2.73872 & -0.59692 \\
\hline C & -1.96464 & -0.78382 & -0.79561 \\
\hline $\mathrm{H}$ & -2.89321 & -0.95314 & -0.26849 \\
\hline C & -3.03993 & 0.95162 & -2.28344 \\
\hline $\mathrm{H}$ & -2.61098 & 1.91891 & -2.00565 \\
\hline $\mathrm{H}$ & -3.92345 & 0.75208 & -1.67676 \\
\hline $\mathrm{H}$ & -3.3383 & 0.94994 & -3.33148 \\
\hline C & -1.05259 & -0.4558 & -2.81843 \\
\hline C & -0.72842 & 0.11149 & -4.14922 \\
\hline $\mathrm{H}$ & 0.28277 & 0.52803 & -4.14301 \\
\hline $\mathrm{H}$ & -1.41195 & 0.91658 & -4.41392 \\
\hline C & 0.23435 & 0.1974 & 2.72269 \\
\hline C & -1.00677 & 0.91332 & 2.48904 \\
\hline C & -2.02895 & -0.04356 & 2.22612 \\
\hline C & -1.44137 & -1.37403 & 2.317 \\
\hline C & -0.0602 & -1.22646 & 2.68318 \\
\hline C & 1.49799 & 0.82067 & 3.22227 \\
\hline $\mathrm{H}$ & 1.67195 & 1.79696 & 2.76615 \\
\hline $\mathrm{H}$ & 2.37058 & 0.18956 & 3.0383 \\
\hline $\mathrm{H}$ & 1.43393 & 0.96607 & 4.30709 \\
\hline C & -1.20346 & 2.38477 & 2.6204 \\
\hline $\mathrm{H}$ & -1.53726 & 2.60427 & 3.64256 \\
\hline $\mathrm{H}$ & -1.96338 & 2.76011 & 1.93259 \\
\hline $\mathrm{H}$ & -0.28222 & 2.93998 & 2.44186 \\
\hline C & -3.48325 & 0.26051 & 2.07604 \\
\hline $\mathrm{H}$ & -3.65485 & 1.21045 & 1.56559 \\
\hline $\mathrm{H}$ & -3.93647 & 0.34009 & 3.072 \\
\hline $\mathrm{H}$ & -4.03011 & -0.5285 & 1.55347 \\
\hline C & -2.2011 & -2.66093 & 2.26975 \\
\hline $\mathrm{H}$ & -1.55679 & -3.51003 & 2.03277 \\
\hline $\mathrm{H}$ & -3.02261 & -2.63979 & 1.549 \\
\hline $\mathrm{H}$ & -2.64705 & -2.85713 & 3.25225 \\
\hline C & 0.85605 & -2.32851 & 3.10036 \\
\hline $\mathrm{H}$ & 0.74562 & -2.49307 & 4.17906 \\
\hline $\mathrm{H}$ & 1.90645 & -2.09178 & 2.92038 \\
\hline $\mathrm{H}$ & 0.62398 & -3.27261 & 2.60265 \\
\hline $\mathrm{H}$ & -0.79154 & -0.66153 & -4.92303 \\
\hline $\mathrm{H}$ & 2.86325 & -0.80717 & -4.28901 \\
\hline $\mathrm{H}$ & 2.28108 & -0.75256 & 1.00186 \\
\hline & 1.58183 & -2.16893 & 0.22568 \\
\hline
\end{tabular}

IrIme_eneTS : $\mathrm{E}=-1153.83811793$

$\begin{array}{lccr}\text { Ir } & -0.22636 & -0.16488 & -0.87472 \\ \mathrm{~N} & -2.49277 & 0.84139 & 2.34713 \\ \mathrm{C} & -1.49102 & -0.13779 & 4.04937 \\ \mathrm{I} & 0.72877 & 1.6805 & 0.85337 \\ \mathrm{~N} & -1.25623 & -0.85118 & 2.87911 \\ \mathrm{C} & -2.26394 & 0.91866 & 3.70796 \\ \mathrm{H} & -2.6789 & 1.70829 & 4.31495 \\ \mathrm{~N} & 0.76211 & -1.83843 & 1.98996 \\ \mathrm{C} & -3.26152 & 1.81168 & 1.57419 \\ \mathrm{H} & -2.62806 & 2.23414 & 0.79187 \\ \mathrm{H} & -3.5825 & 2.60915 & 2.243 \\ \mathrm{H} & -4.14485 & 1.33668 & 1.14134 \\ \mathrm{C} & -1.87654 & -0.23865 & 1.83377 \\ \mathrm{~N} & 2.72111 & -1.17503 & 1.33209 \\ \mathrm{C} & -1.87518 & -0.67323 & 0.43791\end{array}$

\begin{tabular}{|c|c|c|c|}
\hline $\mathrm{C}$ & -0.44499 & -2.04252 & 2.78225 \\
\hline $\mathrm{H}$ & -1.00672 & -2.8532 & 2.31229 \\
\hline $\mathrm{H}$ & -0.17472 & -2.358 & 3.79074 \\
\hline C & 0.8617 & -2.16061 & 0.64187 \\
\hline $\mathrm{H}$ & 0.17422 & -2.84289 & 0.16871 \\
\hline C & 2.10976 & -1.75037 & 0.24786 \\
\hline $\mathrm{H}$ & 2.62348 & -1.8742 & -0.69046 \\
\hline C & 4.03029 & -0.52022 & 1.2986 \\
\hline $\mathrm{H}$ & 3.8873 & 0.56223 & 1.24647 \\
\hline $\mathrm{H}$ & 4.56329 & -0.86399 & 0.41302 \\
\hline $\mathrm{H}$ & 4.609 & -0.7946 & 2.18063 \\
\hline C & 1.8782 & -1.1838 & 2.37969 \\
\hline C & 2.16353 & -0.6656 & 3.73823 \\
\hline $\mathrm{H}$ & 1.2952 & -0.1421 & 4.14184 \\
\hline $\mathrm{H}$ & 2.98671 & 0.04876 & 3.71233 \\
\hline C & -1.14496 & 0.73868 & -2.54992 \\
\hline C & 0.29 & 0.98228 & -2.70724 \\
\hline C & 0.94 & -0.275 & -2.81052 \\
\hline C & -0.08 & -1.32024 & -2.73722 \\
\hline $\mathrm{C}$ & -1.36736 & -0.69717 & -2.65099 \\
\hline $\mathrm{C}$ & -2.2009 & 1.79312 & -2.57297 \\
\hline $\mathrm{H}$ & -1.87337 & 2.70554 & -2.07105 \\
\hline $\mathrm{H}$ & -3.12895 & 1.453 & -2.10899 \\
\hline $\mathrm{H}$ & -2.43 & 2.05128 & -3.61394 \\
\hline C & 0.93 & 2.32167 & -2.83559 \\
\hline $\mathrm{H}$ & 0.95 & 2.60825 & -3.89453 \\
\hline $\mathrm{H}$ & 1.96 & 2.31673 & -2.47072 \\
\hline $\mathrm{H}$ & 0.39 & 3.09288 & -2.28955 \\
\hline C & 2.39 & -0.4817 & -3.10471 \\
\hline $\mathrm{H}$ & 3.02557 & 0.20862 & -2.54332 \\
\hline $\mathrm{H}$ & 2.57665 & -0.2989 & -4.1702 \\
\hline $\mathrm{H}$ & 2.71 & -1.50666 & -2.90849 \\
\hline C & 0.16 & -2.78181 & -2.92739 \\
\hline $\mathrm{H}$ & -0.63 & -3.39309 & -2.49717 \\
\hline $\mathrm{H}$ & 1.11 & -3.10413 & -2.50142 \\
\hline $\mathrm{H}$ & 0.20 & -3.00997 & -3.9997 \\
\hline C & -2.69069 & -1.38025 & -2.7589 \\
\hline $\mathrm{H}$ & -2.96189 & -1.47618 & -3.81699 \\
\hline $\mathrm{H}$ & -3.48955 & -0.81571 & -2.27409 \\
\hline $\mathrm{H}$ & -2.6741 & -2.38644 & -2.33376 \\
\hline $\mathrm{H}$ & 2.43926 & -1.48212 & 4.4171 \\
\hline $\mathrm{H}$ & -1.12926 & -0.46429 & 5.01214 \\
\hline $\mathrm{H}$ & -2.76478 & -0.25657 & -0.03943 \\
\hline $\mathrm{H}$ & -1.99184 & -1.76357 & 0.40687 \\
\hline
\end{tabular}

IrIvy_ene : $\mathrm{E}=-1153.85405564$ 


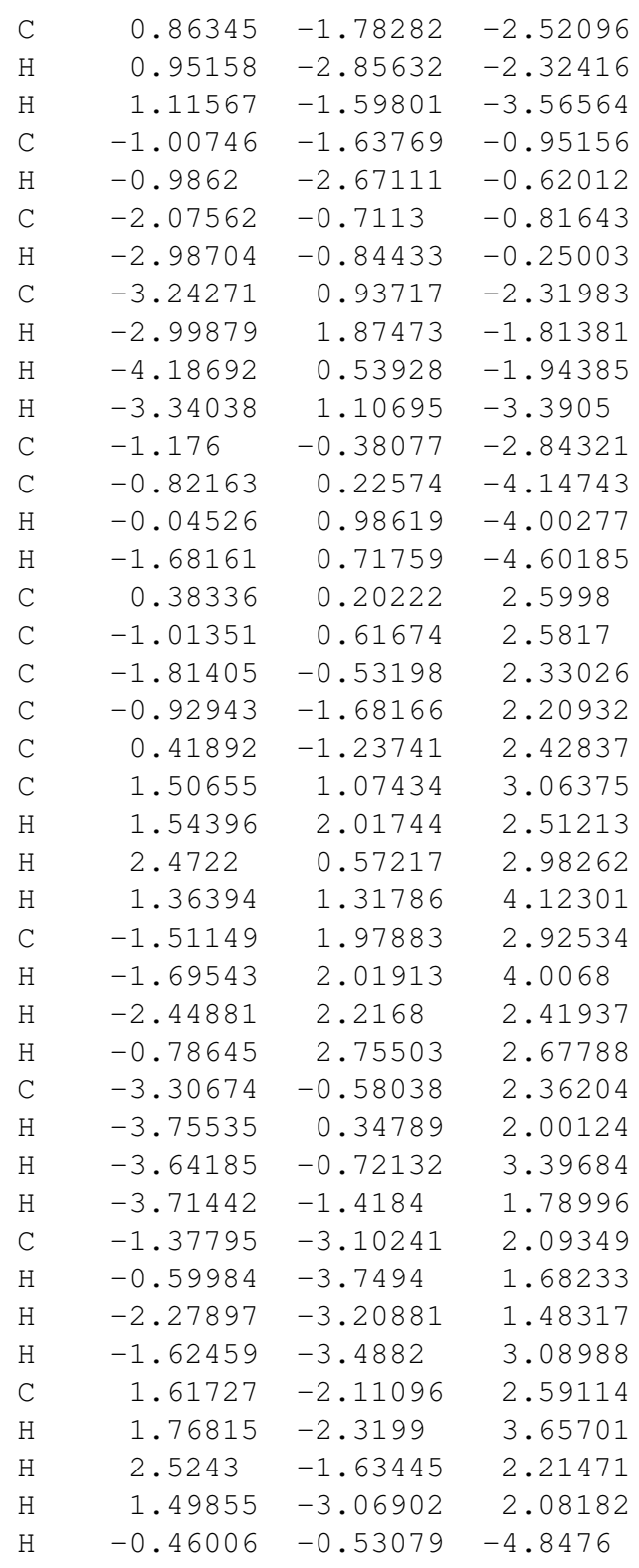

IrIvy_eneTS : $\mathrm{E}=-1153.84388798$

$\begin{array}{lcrl}\text { Ir } & -0.17914 & -0.20654 & 0.75524 \\ \mathrm{~N} & 3.67009 & -0.25117 & -1.06715 \\ \mathrm{C} & 1.5389 & -0.48221 & -0.34933 \\ \mathrm{I} & -0.39342 & 2.12319 & -0.59009 \\ \mathrm{~N} & 1.72638 & -0.98218 & -1.65268 \\ \mathrm{C} & 2.793 & -0.02672 & -0.02265 \\ \mathrm{H} & 3.12039 & 0.50968 & 0.8527 \\ \mathrm{~N} & -0.58986 & -1.19351 & -2.24738 \\ \mathrm{C} & 5.08514 & 0.12346 & -1.06732 \\ \mathrm{H} & 5.71464 & -0.76329 & -1.1639 \\ \mathrm{H} & 5.30759 & 0.61104 & -0.11926 \\ \mathrm{H} & 5.29288 & 0.82421 & -1.87798 \\ \mathrm{C} & 3.01436 & -0.84432 & -2.06505 \\ \mathrm{~N} & -2.42201 & -0.04371 & -2.37882 \\ \mathrm{C} & 3.59153 & -1.30328 & -3.35523 \\ \mathrm{H} & 4.64333 & -1.02275 & -3.42106 \\ \mathrm{H} & 3.08468 & -0.84764 & -4.213 \\ \mathrm{H} & 3.53734 & -2.39242 & -3.46191 \\ \mathrm{C} & 0.74492 & -1.70887 & -2.44384 \\ \mathrm{H} & 0.73153 & -2.76489 & -2.15786 \\ \mathrm{H} & 1.00587 & -1.64521 & -3.50033\end{array}$

$\begin{array}{llll}\mathrm{C} & -1.36859 & -1.55929 & -1.15306 \\ \mathrm{H} & -1.17199 & -2.4693 & -0.60799 \\ \mathrm{C} & -2.53583 & -0.84376 & -1.27775 \\ \mathrm{H} & -3.4334 & -0.86602 & -0.68091 \\ \mathrm{C} & -3.41751 & 0.94625 & -2.79827 \\ \mathrm{H} & -3.09126 & 1.93882 & -2.47777 \\ \mathrm{H} & -4.36624 & 0.70039 & -2.32292 \\ \mathrm{H} & -3.54744 & 0.90838 & -3.8799 \\ \mathrm{C} & -1.20781 & -0.21769 & -2.94055 \\ \mathrm{C} & -0.68923 & 0.47292 & -4.14421 \\ \mathrm{H} & 0.37674 & 0.68223 & -4.03666 \\ \mathrm{H} & -1.19032 & 1.43197 & -4.27999 \\ \mathrm{C} & 0.36017 & 0.25277 & 2.75897 \\ \mathrm{C} & -1.06771 & 0.57622 & 2.6621 \\ \mathrm{C} & -1.7578 & -0.61431 & 2.34046 \\ \mathrm{C} & -0.78463 & -1.70788 & 2.25775 \\ \mathrm{C} & 0.50608 & -1.18705 & 2.58361 \\ \mathrm{C} & 1.40907 & 1.19069 & 3.25482 \\ \mathrm{H} & 1.31805 & 2.18048 & 2.80317 \\ \mathrm{H} & 2.4167 & 0.81148 & 3.0747 \\ \mathrm{H} & 1.30008 & 1.30776 & 4.34064 \\ \mathrm{C} & -1.66851 & 1.91159 & 2.94627 \\ \mathrm{H} & -1.88352 & 1.98714 & 4.01935 \\ \mathrm{H} & -2.6043 & 2.06241 & 2.40528 \\ \mathrm{H} & -0.99345 & 2.7265 & 2.68041 \\ \mathrm{C} & -3.24085 & -0.76871 & 2.24495 \\ \mathrm{H} & -3.72306 & 0.13764 & 1.87248 \\ \mathrm{H} & -3.64853 & -0.96518 & 3.24413 \\ \mathrm{H} & -3.53455 & -1.61466 & 1.61833 \\ \mathrm{C} & -1.12805 & -3.153 & 2.09079 \\ \mathrm{H} & -0.3006 & -3.72629 & 1.66618 \\ \mathrm{H} & -2.01485 & -3.30328 & 1.46933 \\ \mathrm{H} & -1.35577 & -3.59054 & 3.07052 \\ \mathrm{C} & 1.75266 & -1.97398 & 2.81212 \\ \mathrm{H} & 1.86212 & -2.17423 & 3.88463 \\ \mathrm{H} & 2.64426 & -1.43548 & 2.48467 \\ \mathrm{H} & 1.73001 & -2.93565 & 2.29618 \\ \mathrm{H} & -0.85002 & -0.13201 & -5.04508\end{array}$

TS2mevy : $\mathrm{E}=-1153.81572305$

$\begin{array}{lccc}\text { Ir } & -0.08209 & -0.35563 & -0.79807 \\ \mathrm{~N} & -2.50212 & -0.51182 & 2.58819 \\ \mathrm{C} & -0.85106 & -0.67902 & 4.04283 \\ \mathrm{I} & -0.74697 & 1.91661 & 0.59907 \\ \mathrm{~N} & -0.45321 & -1.16363 & 2.80632 \\ \mathrm{C} & -2.1346 & -0.27173 & 3.89958 \\ \mathrm{H} & -2.81835 & 0.1555 & 4.61656 \\ \mathrm{~N} & 1.7365 & -0.76088 & 1.8337 \\ \mathrm{C} & -3.80558 & -0.17716 & 2.01839 \\ \mathrm{H} & -3.68356 & 0.61094 & 1.27158 \\ \mathrm{H} & -4.44744 & 0.18472 & 2.82052 \\ \mathrm{H} & -4.26248 & -1.06415 & 1.57609 \\ \mathrm{C} & -1.46775 & -1.04194 & 1.9153 \\ \mathrm{~N} & 3.48314 & 0.46178 & 1.46482 \\ \mathrm{C} & -1.40626 & -1.44053 & 0.50404 \\ \mathrm{C} & 0.83941 & -1.71269 & 2.47601 \\ \mathrm{H} & 0.70523 & -2.56269 & 1.80373 \\ \mathrm{H} & 1.30674 & -2.07885 & 3.38955 \\ \mathrm{C} & 1.63222 & -0.3397 & 0.49609 \\ \mathrm{H} & 1.11639 & -1.35966 & -0.25476 \\ \mathrm{C} & 2.76731 & 0.40335 & 0.29005 \\ \mathrm{H} & 3.08821 & 0.94042 & -0.58725 \\ \mathrm{C} & 4.7323 & 1.20844 & 1.64566 \\ \mathrm{H} & 4.59018 & 2.00851 & 2.37459\end{array}$




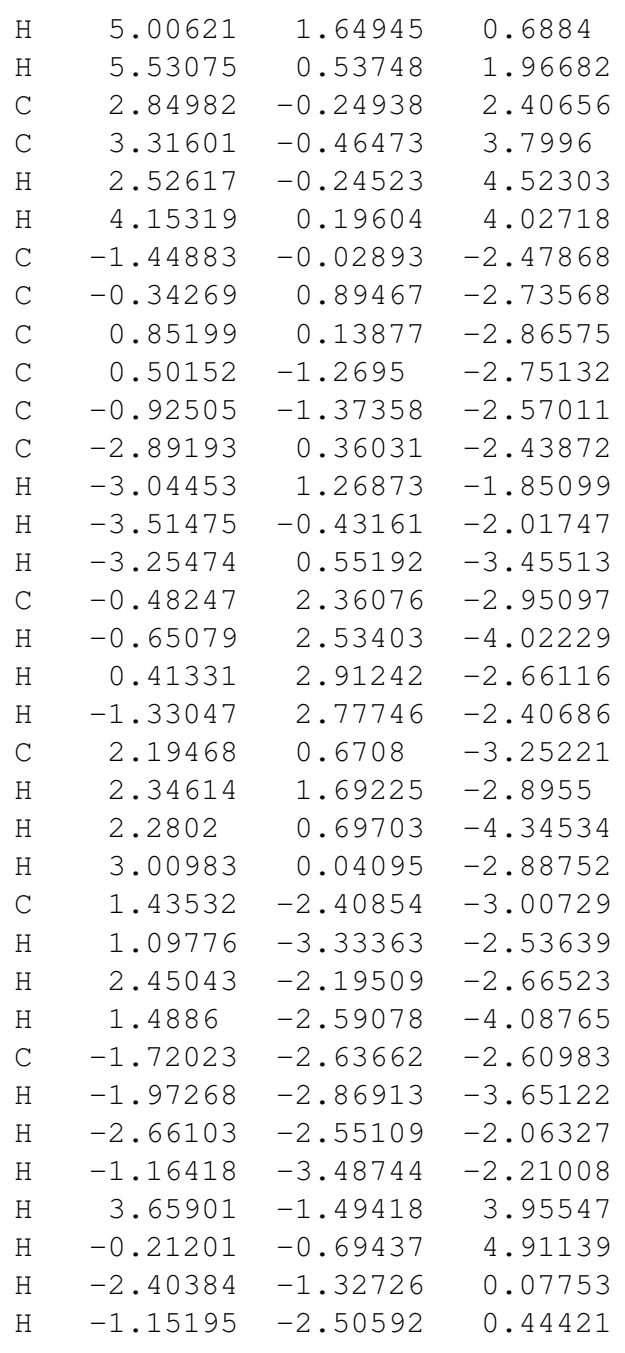

\section{TS2vyvy : E $=-1153.81583697$}

$\begin{array}{lccc}\text { Ir } & 0.61396 & 0.00217 & 0.46673 \\ \text { I } & 1.55528 & 0.89451 & -1.92564 \\ \text { N } & -2.29921 & 1.01799 & 0.46741 \\ \text { C } & 0.88989 & -0.48846 & 2.6518 \\ \text { C } & 1.96305 & 0.42278 & 2.26059 \\ \text { C } & 2.74436 & -0.21385 & 1.24988 \\ \text { C } & -0.02114 & -0.2777 & 3.81878 \\ \text { H } & -0.87802 & -0.95492 & 3.80303 \\ \text { H } & -0.38894 & 0.74968 & 3.87665 \\ \text { H } & 0.52455 & -0.47688 & 4.74914 \\ \text { C } & 2.2943 & 1.71363 & 2.94044 \\ \text { H } & 2.79298 & 2.41223 & 2.26613 \\ \text { H } & 2.97826 & 1.51508 & 3.7743 \\ \text { H } & 1.41031 & 2.20265 & 3.35538 \\ \text { C } & 4.0202 & 0.29819 & 0.67596 \\ \text { H } & 4.84522 & -0.02569 & 1.32344 \\ \text { H } & 4.04066 & 1.38783 & 0.62407 \\ \text { H } & 4.20313 & -0.09069 & -0.32608 \\ \text { C } & -0.9811 & 1.43374 & 0.23048 \\ \text { C } & -3.17774 & 1.96018 & 0.05223 \\ \text { C } & -2.61077 & -0.3659 & 0.78914 \\ \text { H } & -3.68779 & -0.49255 & 0.87808 \\ \text { H } & -2.12948 & -0.61588 & 1.73642 \\ \text { N } & -2.0741 & -1.22019 & -0.25342 \\ \text { C } & 1.03666 & -1.68937 & 1.8829 \\ \mathrm{C} & 2.13902 & -1.50233 & 0.95504 \\ & & & \end{array}$

$\begin{array}{llll}\mathrm{C} & 0.27411 & -2.95879 & 2.06821 \\ \mathrm{H} & 0.11638 & -3.48072 & 1.12242 \\ \mathrm{H} & 0.85026 & -3.62641 & 2.72003 \\ \mathrm{H} & -0.69438 & -2.79603 & 2.54577 \\ \mathrm{C} & 2.73317 & -2.54604 & 0.06489 \\ \mathrm{H} & 3.54446 & -3.06451 & 0.58896 \\ \mathrm{H} & 1.99528 & -3.29672 & -0.22429 \\ \mathrm{H} & 3.14878 & -2.10465 & -0.84345 \\ \mathrm{C} & -0.71414 & -1.16648 & -0.58561 \\ \mathrm{C} & -2.75005 & -2.06328 & -1.0728 \\ \mathrm{~N} & -2.45485 & 2.98721 & -0.41385 \\ \mathrm{~N} & -1.84561 & -2.56007 & -1.92418 \\ \mathrm{C} & -1.11429 & 2.68277 & -0.32076 \\ \mathrm{C} & -0.6006 & -2.02031 & -1.6475 \\ \mathrm{H} & -0.35093 & 3.36872 & -0.65589 \\ \mathrm{H} & 0.25763 & -2.25645 & -2.25689 \\ \mathrm{C} & -2.12146 & -3.53368 & -2.98172 \\ \mathrm{H} & -2.28096 & -4.52662 & -2.55437 \\ \mathrm{H} & -2.9985 & -3.22979 & -3.55487 \\ \mathrm{H} & -1.26195 & -3.56567 & -3.65013 \\ \mathrm{C} & -2.9888 & 4.25258 & -0.92873 \\ \mathrm{H} & -3.70338 & 4.06089 & -1.73036 \\ \mathrm{H} & -3.4631 & 4.81826 & -0.12399 \\ \mathrm{H} & -2.15908 & 4.8326 & -1.33021 \\ \mathrm{C} & -4.19589 & -2.40515 & -1.05935 \\ \mathrm{H} & -4.33491 & -3.47905 & -1.2121 \\ \mathrm{H} & -4.66454 & -2.15777 & -0.10573 \\ \mathrm{H} & -4.73976 & -1.88562 & -1.85701 \\ \mathrm{C} & -4.65961 & 1.88122 & 0.09642 \\ \mathrm{H} & -5.00626 & 1.43547 & 1.03228 \\ \mathrm{H} & -5.09585 & 2.87962 & 0.03403 \\ \mathrm{H} & -5.0577 & 1.296 & -0.74059 \\ \mathrm{H} & 0.24423 & 1.53489 & 0.88286 \\ & & & \end{array}$

\section{$\operatorname{IrIvyvyH}^{+}: \mathrm{E}=-1153.83441750$}

$\begin{array}{lccr}\text { Ir } & 0.67019 & -0.2178 & -0.20149 \\ \mathrm{I} & 0.94582 & 0.13503 & 2.50713 \\ \mathrm{~N} & -2.27829 & -1.01701 & -0.54925 \\ \mathrm{C} & 1.64091 & -1.18432 & -1.95898 \\ \mathrm{C} & 2.63432 & -1.09468 & -0.90913 \\ \mathrm{C} & 2.89358 & 0.30968 & -0.64584 \\ \mathrm{C} & 1.21721 & -2.44102 & -2.64531 \\ \mathrm{H} & 0.24003 & -2.33869 & -3.12062 \\ \mathrm{H} & 1.17405 & -3.28587 & -1.95535 \\ \mathrm{H} & 1.94313 & -2.68881 & -3.42881 \\ \mathrm{C} & 3.44507 & -2.22387 & -0.3591 \\ \mathrm{H} & 3.71133 & -2.06168 & 0.68725 \\ \mathrm{H} & 4.37791 & -2.29737 & -0.93092 \\ \mathrm{H} & 2.92934 & -3.18187 & -0.44294 \\ \mathrm{C} & 3.94678 & 0.823 & 0.27621 \\ \mathrm{H} & 4.92295 & 0.74544 & -0.21827 \\ \mathrm{H} & 3.99215 & 0.24698 & 1.20272 \\ \mathrm{H} & 3.78827 & 1.8698 & 0.53826 \\ \mathrm{C} & -1.02098 & -1.36706 & -0.04845 \\ \mathrm{C} & -3.21603 & -1.94345 & -0.23331 \\ \mathrm{C} & -2.50896 & 0.19229 & -1.31025 \\ \mathrm{H} & -3.5765 & 0.32437 & -1.48145 \\ \mathrm{H} & -2.00017 & 0.1166 & -2.27463 \\ \mathrm{~N} & -1.98214 & 1.3284 & -0.58184 \\ \mathrm{C} & 1.2611 & 0.15893 & -2.31562 \\ \mathrm{C} & 2.05373 & 1.07771 & -1.49946 \\ \mathrm{C} & 0.44334 & 0.55763 & -3.50163 \\ \mathrm{H} & -0.11875 & 1.47762 & -3.32399 \\ \mathrm{H} & 1.10816 & 0.74706 & -4.35325\end{array}$




$\begin{array}{lrcl}\mathrm{H} & -0.24659 & -0.23165 & -3.80806 \\ \mathrm{C} & 2.09411 & 2.55932 & -1.66722 \\ \mathrm{H} & 2.82256 & 2.8109 & -2.44767 \\ \mathrm{H} & 1.12822 & 2.96415 & -1.97402 \\ \mathrm{H} & 2.40789 & 3.06504 & -0.75236 \\ \mathrm{C} & -0.67772 & 1.35938 & -0.08179 \\ \mathrm{C} & -2.65237 & 2.46839 & -0.29521 \\ \mathrm{~N} & -2.58683 & -2.89358 & 0.46376 \\ \mathrm{~N} & -1.79762 & 3.24705 & 0.37983 \\ \mathrm{C} & -1.25094 & -2.55779 & 0.59038 \\ \mathrm{C} & -0.59329 & 2.58522 & 0.5233 \\ \mathrm{H} & -0.57977 & -3.18033 & 1.16126 \\ \mathrm{H} & 0.21567 & 3.02379 & 1.08573 \\ \mathrm{C} & -2.08521 & 4.58184 & 0.91065 \\ \mathrm{H} & -2.31486 & 5.27402 & 0.09818 \\ \mathrm{H} & -2.91648 & 4.53806 & 1.6165 \\ \mathrm{H} & -1.19943 & 4.93609 & 1.43599 \\ \mathrm{C} & -3.19983 & -4.09795 & 1.02854 \\ \mathrm{H} & -3.97302 & -3.82733 & 1.74992 \\ \mathrm{H} & -3.62193 & -4.71837 & 0.23573 \\ \mathrm{H} & -2.42462 & -4.66393 & 1.54305 \\ \mathrm{C} & -4.05276 & 2.79925 & -0.66408 \\ \mathrm{H} & -4.21996 & 2.70463 & -1.74171 \\ \mathrm{H} & -4.76897 & 2.15506 & -0.14205 \\ \mathrm{H} & -4.28601 & 3.8289 & -0.39054 \\ \mathrm{C} & -4.65382 & -1.91884 & -0.6055 \\ \mathrm{H} & -4.78985 & -1.90057 & -1.69192 \\ \mathrm{H} & -5.15947 & -2.80896 & -0.22964 \\ \mathrm{H} & -5.16584 & -1.0512 & -0.17565 \\ \mathrm{H} & 1.07186 & -1.49368 & 0.64975\end{array}$

IrIvyvy : $\mathrm{E}=-1153.43202156$

$\begin{array}{lrcc}\text { Ir } & -0.60542 & -0.00026 & 0.38059 \\ \text { I } & -1.23786 & 0.0003 & -2.29332 \\ \text { N } & 2.18078 & -1.1841 & 0.20753 \\ \text { C } & -1.00347 & -0.00023 & 2.52609 \\ \text { C } & -1.66876 & -1.17189 & 1.97496 \\ \mathrm{C} & -2.64747 & -0.73381 & 1.04427 \\ \mathrm{C} & -0.07382 & 0.00065 & 3.70049 \\ \mathrm{H} & 0.56528 & 0.88808 & 3.70908 \\ \mathrm{H} & 0.56718 & -0.88542 & 3.70894 \\ \mathrm{H} & -0.63803 & -0.00003 & 4.64185 \\ \mathrm{C} & -1.40058 & -2.58544 & 2.38231 \\ \mathrm{H} & -1.65725 & -3.28934 & 1.58792 \\ \mathrm{H} & -2.00078 & -2.84343 & 3.26295 \\ \mathrm{H} & -0.34993 & -2.73943 & 2.64014 \\ \mathrm{C} & -3.62172 & -1.58123 & 0.2915 \\ \mathrm{H} & -4.60195 & -1.55573 & 0.78291 \\ \mathrm{H} & -3.29973 & -2.62381 & 0.24627 \\ \mathrm{H} & -3.74554 & -1.22632 & -0.73479 \\ \mathrm{C} & 0.81577 & -1.36989 & -0.05718 \\ \mathrm{C} & 2.9323 & -2.22206 & -0.22803 \\ \mathrm{C} & 2.66026 & 0.00049 & 0.88781 \\ \mathrm{H} & 3.74983 & 0.0008 & 0.91721 \\ \mathrm{H} & 2.26416 & 0.00002 & 1.9076 \\ \mathrm{~N} & 2.18009 & 1.18526 & 0.20831 \\ \mathrm{C} & -1.67051 & 1.17031 & 1.97462 \\ \mathrm{C} & -2.64857 & 0.73048 & 1.04411 \\ \mathrm{C} & -1.40433 & 2.58437 & 2.38153 \\ \mathrm{H} & -1.66275 & 3.28769 & 1.58719 \\ \mathrm{H} & -2.00427 & 2.84158 & 3.26258 \\ \mathrm{H} & -0.35373 & 2.74012 & 2.63848 \\ \mathrm{C} & -3.62408 & 1.57621 & 0.2911 \\ \mathrm{H} & -4.60392 & 1.55037 & 0.78328\end{array}$

$\begin{array}{cccc}\mathrm{H} & -3.30305 & 2.61901 & 0.24442 \\ \mathrm{H} & -3.74833 & 1.22004 & -0.73469 \\ \mathrm{C} & 0.81498 & 1.37048 & -0.05636 \\ \mathrm{C} & 2.93106 & 2.22394 & -0.22645 \\ \mathrm{~N} & 2.07654 & -3.09549 & -0.77181 \\ \mathrm{~N} & 2.07487 & 3.09729 & -0.76965 \\ \mathrm{C} & 0.78789 & -2.59504 & -0.67944 \\ \mathrm{C} & 0.78649 & 2.59603 & -0.67781 \\ \mathrm{H} & -0.04528 & -3.11995 & -1.11849 \\ \mathrm{H} & -0.04694 & 3.12089 & -1.11645 \\ \mathrm{C} & 2.42576 & 4.37316 & -1.38207 \\ \mathrm{H} & 2.84948 & 5.05535 & -0.64132 \\ \mathrm{H} & 3.13314 & 4.22503 & -2.20058 \\ \mathrm{H} & 1.51623 & 4.81513 & -1.7868 \\ \mathrm{C} & 2.42788 & -4.37116 & -1.38438 \\ \mathrm{H} & 3.13984 & -4.22324 & -2.19892 \\ \mathrm{H} & 2.84629 & -5.05545 & -0.64255 \\ \mathrm{H} & 1.51957 & -4.81042 & -1.79478 \\ \mathrm{C} & 4.40348 & 2.37892 & -0.10225 \\ \mathrm{H} & 4.7279 & 2.3463 & 0.94358 \\ \mathrm{H} & 4.94076 & 1.59811 & -0.65223 \\ \mathrm{H} & 4.72182 & 3.33916 & -0.51026 \\ \mathrm{C} & 4.4047 & -2.37674 & -0.10331 \\ \mathrm{H} & 4.94194 & -1.59276 & -0.64873 \\ \mathrm{H} & 4.72821 & -2.34931 & 0.94298 \\ \mathrm{H} & 4.72404 & -3.33465 & -0.51599\end{array}$

\section{bisImme $^{+}: \mathrm{E}=-648.000285451$}

$\mathrm{N}, 0,-3.1250737543,0.7690230303,-0.5237418888$ C, $0,-1.9580235395,-1.0569892707,-1.023327329$ $\mathrm{N}, 0,-1.3410745765,-0.3871160035,0.0567318015$ $\mathrm{C}, 0,-3.0422385404,-0.3381488294,-1.3587273566$ $\mathrm{N}, 0,1.0501881922,-0.323992657,0.3803332562$ C, $0,-4.1354955386,1.7987233962,-0.5498285287$ $\mathrm{H}, 0,-4.6866013865,1.8281673764,0.396744976$ $\mathrm{H}, \mathrm{O},-4.8377567991,1.5898262011,-1.3575107826$ $\mathrm{H}, \mathrm{O},-3.6810304199,2.7810709927,-0.7224393308$ C, $0,-2.0711458805,0.7733293907,0.3730869756$ C, $0,-1.7846775349,1.6990812473,1.3222224994$ $\mathrm{H}, \mathrm{O},-2.4655986443,2.5154547322,1.5161837143$ $\mathrm{H}, 0,-0.9324334579,1.5954394552,1.9814646362$ $C, 0,-0.2723733434,-0.8658441694,0.8316653146$ C, $0,2.2172351939,-0.9904083434,0.3467159868$ C, $0,1.2428941063,0.9725569167,-0.0489928447$ $\mathrm{H}, 0,-1.561466549,-1.9718764338,-1.4324489641$ $\mathrm{H}, 0,-3.7752371856,-0.5151862991,-2.1305124714$ $\mathrm{H}, 0,-0.4040630728,-0.5712385395,1.8787869656$ $\mathrm{H}, 0,-0.2055946367,-1.9538854703,0.7701387154$ $C, 0,2.5622662227,1.090872008,-0.3511075164$ $\mathrm{N}, 0,3.152664652,-0.1323438655,-0.1010695667$ $\mathrm{H}, 0,0.4276975891,1.6835232074,-0.0747396377$ $\mathrm{H}, 0,3.127988673,1.9331914776,-0.7168963287$ C, $0,2.4351485967,-2.4083720868,0.7316460021$ $\mathrm{H}, 0,2.1936734421,-2.5752759914,1.7861157288$ $\mathrm{H}, 0,1.8164928002,-3.0811504132,0.1287666897$ $\mathrm{H}, 0,3.4763006032,-2.6936585288,0.578945913$ C, $0,4.5681514912,-0.4341890874,-0.3069412687$ $\mathrm{H}, 0,5.0276296267,-0.7599516806,0.6281562057$ $\mathrm{H}, \mathrm{O}, 4.6876409697,-1.2025955318,-1.0733333606$ $\mathrm{H}, 0,5.0649794537,0.4754848857,-0.6416694915$

\section{bisImvy $^{+}: \mathrm{E}=-647.978760221$}

$\mathrm{N}, 0,-0.7419965502,-0.2483326479,-2.9448873439$ C, $0,-1.3897329088,0.6479767901,-0.9390662042$ 
$\mathrm{N}, 0,0.006969746,0.5722261089,-1.1155718712$ C, $0,-1.8182108485,0.1078305118,-2.1287353331$ $\mathrm{N}, 0,0.7890992454,0.2652303972,1.145462393$ $C, 0,-0.8502452064,-0.8180470842,-4.2813574068$ $\mathrm{H}, \mathrm{O},-0.4345868641,-0.1388078674,-5.0298221571$ $\mathrm{H}, 0,-1.9070546537,-0.9698602799,-4.4989920541$ $\mathrm{H}, 0,-0.3417360255,-1.783426942,-4.3359485276$ C, $0,0.3954497011,0.0417469001,-2.3063115234$ C, $0,1.7780998221,-0.1585543542,-2.8182004021$ $\mathrm{H}, \mathrm{O}, 1.7578354494,-0.5225287137,-3.8468926349$ $\mathrm{H}, \mathrm{O}, 2.3345884308,-0.8955582282,-2.2275447632$ $\mathrm{H}, 0,2.3487289971,0.7765908533,-2.8204748676$ C, $0,0.8882302004,1.0565161026,-0.0886520565$ C, $0,-0.1327616222,0.445076004,2.1162306067$ C, $0,1.6025515125,-0.8024806839,1.4729563652$ $\mathrm{H}, \mathrm{O},-2.8227039207,-0.0531002047,-2.493797502$ $\mathrm{H}, 0,1.9304985439,1.0280903578,-0.410688334$ $\mathrm{H}, 0,0.6216642401,2.0854782825,0.156679261$ C, $0,-1.2133405139,1.4510927759,2.1042768564$ $\mathrm{H}, \mathrm{O},-0.8181481642,2.4603559444,2.2677944043$ $\mathrm{H}, 0,-1.6735837102,1.3918491565,1.091425739$ $\mathrm{H}, \mathrm{O},-1.9531641977,1.2411961038,2.8772985613$ $\mathrm{N}, 0,0.0978803298,-0.4938518712,3.0496058615$ C, $0,1.1691755631,-1.2787087974,2.6662224916$ $\mathrm{H}, \mathrm{O}, 2.4169148065,-1.1225654294,0.8428591641$ $\mathrm{H}, 0,1.528917746,-2.0940079664,3.2737418375$ C, $0,-0.6746594044,-0.6742730388,4.2770994447$ $\mathrm{H}, 0,-0.7268784955,0.2649697424,4.829486619$ $\mathrm{H}, 0,-1.68145694,-1.0264233222,4.0427675036$ $\mathrm{H}, \mathrm{O},-0.170842409,-1.4182061518,4.8930674437$

\section{bisImvycyc $^{+}: \mathrm{E}=-648.007115663$}

$\begin{array}{lrrr}\text { N } & 2.2248 & 0.8667 & -0.06856 \\ \mathrm{C} & 0.0958 & 0.4143 & 0.30255 \\ \mathrm{~N} & 0.8207 & -0.7417 & 0.15923 \\ \mathrm{C} & 0.9822 & 1.4395 & 0.15955 \\ \mathrm{~N} & -1.3706 & -1.4112 & 0.24776 \\ \mathrm{C} & 3.4505 & 1.6102 & -0.34882 \\ \mathrm{H} & 4.3055 & 1.0934 & 0.08823 \\ \mathrm{H} & 3.3713 & 2.5979 & 0.10519 \\ \mathrm{H} & 3.5958 & 1.7167 & -1.42678 \\ \mathrm{C} & 2.1122 & -0.4781 & -0.05948 \\ \mathrm{C} & 3.2071 & -1.4590 & -0.26084 \\ \mathrm{H} & 3.7036 & -1.3060 & -1.22404 \\ \mathrm{H} & 2.8110 & -2.4753 & -0.24305 \\ \mathrm{H} & 3.9623 & -1.3783 & 0.52818 \\ \mathrm{C} & -0.0427 & -1.9347 & 0.40755 \\ \mathrm{C} & -1.3745 & 0.0433 & 0.60347 \\ \mathrm{C} & -1.9263 & -1.4742 & -1.07468 \\ \mathrm{H} & 0.8578 & 2.5097 & 0.20189 \\ \mathrm{H} & 0.1682 & -2.7339 & -0.30433 \\ \mathrm{H} & 0.1502 & -2.2788 & 1.42878 \\ \mathrm{C} & -1.7216 & 0.3009 & 2.06436 \\ \mathrm{H} & -1.0172 & -0.2048 & 2.73158 \\ \mathrm{H} & -1.6882 & 1.3700 & 2.29282 \\ \mathrm{H} & -2.7277 & -0.0752 & 2.25691 \\ \mathrm{~N} & -2.3511 & 0.6056 & -0.31884 \\ \mathrm{C} & -2.4925 & -0.3027 & -1.37577 \\ \mathrm{H} & -2.0010 & -2.4231 & -1.58540 \\ \mathrm{H} & -3.0976 & -0.0481 & -2.23494 \\ \mathrm{C} & -2.3686 & 2.0324 & -0.56282 \\ \mathrm{H} & -2.4704 & 2.5746 & 0.38092 \\ \mathrm{H} & -1.4752 & 2.3987 & -1.09424 \\ \mathrm{H} & -3.2444 & 2.2742 & -1.16927\end{array}$

\section{bisImvycycTS $^{+}: \mathrm{E}=-647.975771900$}

$\begin{array}{lcrc}\mathrm{N} & -2.85919 & -0.72074 & -0.35556 \\ \mathrm{C} & -0.64522 & -1.15986 & 0.04489 \\ \mathrm{~N} & -1.03926 & 0.15331 & 0.35389 \\ \mathrm{C} & -1.84021 & -1.67297 & -0.40611 \\ \mathrm{~N} & 1.19656 & 0.92996 & 0.30911 \\ \mathrm{C} & -4.2413 & -0.93352 & -0.76257 \\ \mathrm{H} & -4.92493 & -0.74875 & 0.06936 \\ \mathrm{H} & -4.34565 & -1.97256 & -1.0739 \\ \mathrm{H} & -4.50496 & -0.28967 & -1.60554 \\ \mathrm{C} & -2.34964 & 0.42124 & 0.12009 \\ \mathrm{C} & -3.08934 & 1.68773 & 0.36811 \\ \mathrm{H} & -4.06256 & 1.66854 & -0.12584 \\ \mathrm{H} & -2.54964 & 2.55576 & -0.02484 \\ \mathrm{H} & -3.26643 & 1.85816 & 1.43685 \\ \mathrm{C} & -0.09967 & 1.08967 & 0.94267 \\ \mathrm{C} & 2.04098 & -0.09439 & 0.55747 \\ \mathrm{C} & 1.56649 & 1.55382 & -0.86863 \\ \mathrm{H} & -2.07271 & -2.66299 & -0.77258 \\ \mathrm{H} & -0.42501 & 2.12209 & 0.80214 \\ \mathrm{H} & 0.01179 & 0.90789 & 2.01456 \\ \mathrm{C} & 2.04124 & -1.0218 & 1.71338 \\ \mathrm{H} & 1.48914 & -0.59698 & 2.55286 \\ \mathrm{H} & 1.53226 & -1.94404 & 1.42133 \\ \mathrm{H} & 3.06291 & -1.22424 & 2.04603 \\ \mathrm{~N} & 2.96142 & -0.08689 & -0.41786 \\ \mathrm{C} & 2.67904 & 0.92574 & -1.31687 \\ \mathrm{H} & 1.0123 & 2.38355 & -1.2774 \\ \mathrm{H} & 3.29645 & 1.11191 & -2.18121 \\ \mathrm{C} & 4.0793 & -1.01962 & -0.52728 \\ \mathrm{H} & 4.82266 & -0.82054 & 0.24813 \\ \mathrm{H} & 3.71589 & -2.04495 & -0.44296 \\ \mathrm{H} & 4.54176 & -0.88887 & -1.50488\end{array}$

\section{IrImemeEt : $\mathrm{E}=-1192.69939857$}

$\begin{array}{lccl}\text { Ir } & 0.04283 & -0.30287 & -0.89947 \\ \mathrm{~N} & -2.8592 & 0.37203 & 1.48908 \\ \mathrm{C} & -2.38414 & -0.0174 & 3.5961 \\ \mathrm{I} & 0.12027 & 1.96157 & 0.73121 \\ \mathrm{~N} & -1.70812 & -0.92362 & 2.78835 \\ \mathrm{C} & -3.09801 & 0.78961 & 2.78148 \\ \mathrm{H} & -3.73506 & 1.63073 & 3.00233 \\ \mathrm{~N} & 1.26716 & -1.3872 & 2.76205 \\ \mathrm{C} & -3.42234 & 0.97695 & 0.29648 \\ \mathrm{H} & -2.62192 & 1.11066 & -0.43438 \\ \mathrm{H} & -3.83219 & 1.9515 & 0.55946 \\ \mathrm{H} & -4.21659 & 0.34493 & -0.11219 \\ \mathrm{C} & -1.99713 & -0.66773 & 1.48289 \\ \mathrm{~N} & 2.75703 & -0.01702 & 1.99589 \\ \mathrm{C} & -1.49289 & -1.32227 & 0.28069 \\ \mathrm{C} & 0.31678 & -2.47761 & 2.8293 \\ \mathrm{H} & 0.24789 & -2.91135 & 1.83457 \\ \mathrm{H} & 0.71379 & -3.25687 & 3.4898 \\ \mathrm{C} & 1.82092 & -0.73718 & 3.85449 \\ \mathrm{H} & 1.51957 & -0.94734 & 4.868 \\ \mathrm{C} & 2.74664 & 0.12259 & 3.36919 \\ \mathrm{H} & 3.39334 & 0.82014 & 3.87681 \\ \mathrm{C} & 3.6171 & 0.71565 & 1.07979 \\ \mathrm{H} & 4.35608 & 0.04357 & 0.63442 \\ \mathrm{H} & 4.13246 & 1.49596 & 1.63884 \\ \mathrm{H} & 3.00008 & 1.18209 & 0.3086 \\ \mathrm{C} & 1.83998 & -0.93254 & 1.61836 \\ \mathrm{C} & 1.55035 & -1.34696 & 0.2521 \\ \mathrm{H} & 1.28116 & -2.4083 & 0.23891\end{array}$




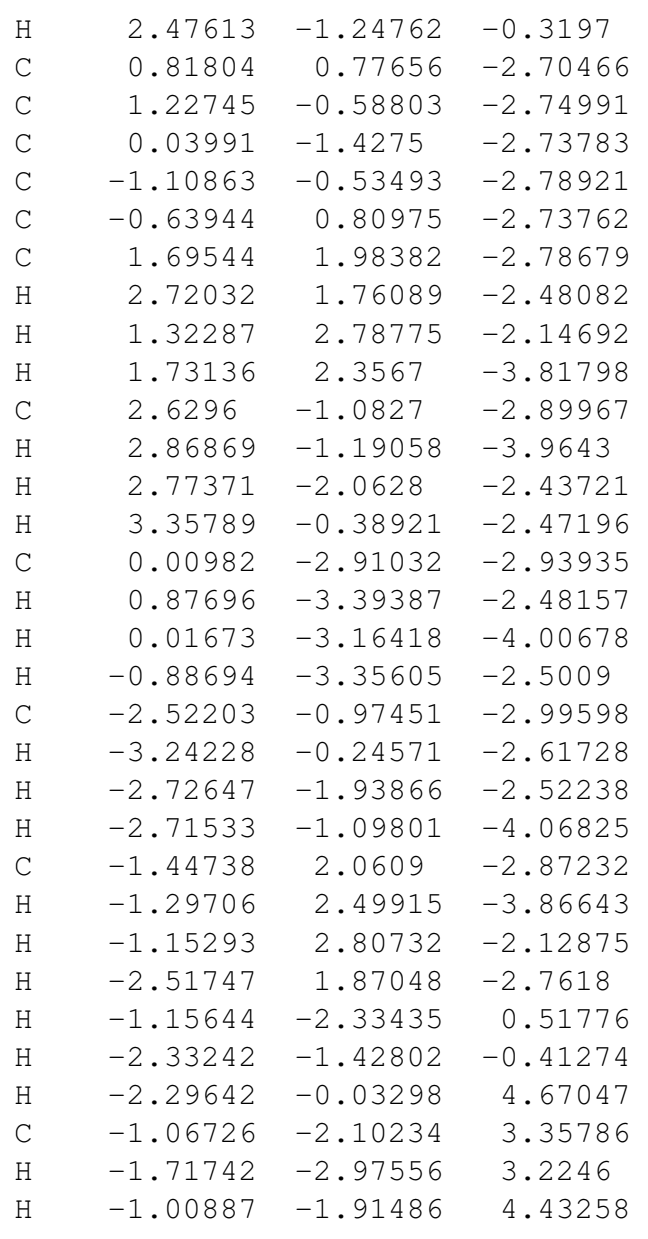

IrImevyEt : $\mathrm{E}=-1192.71158172$

$\begin{array}{lrrr}\text { Ir } & -0.02265 & 0.28339 & 0.75877 \\ \mathrm{~N} & -2.09276 & -1.66205 & -2.39806 \\ \mathrm{C} & -0.96731 & -0.32064 & -0.96092 \\ \mathrm{I} & 1.36333 & -2.12938 & 0.76383 \\ \mathrm{~N} & -1.51495 & 0.39433 & -2.05459 \\ \mathrm{C} & -1.36058 & -1.61431 & -1.22999 \\ \mathrm{H} & -1.13754 & -2.51392 & -0.67867 \\ \mathrm{~N} & 0.99363 & 1.66287 & -2.52341 \\ \mathrm{C} & -2.65634 & -2.87692 & -2.97252 \\ \mathrm{H} & -3.74678 & -2.81948 & -3.00874 \\ \mathrm{H} & -2.37038 & -3.71405 & -2.33669 \\ \mathrm{H} & -2.26119 & -3.0505 & -3.97582 \\ \mathrm{C} & -2.1853 & -0.42854 & -2.90152 \\ \mathrm{~N} & 2.63678 & 0.25761 & -2.42327 \\ \mathrm{C} & -2.91005 & -0.05219 & -4.14435 \\ \mathrm{H} & -3.3814 & -0.93212 & -4.58433 \\ \mathrm{H} & -2.23826 & 0.3676 & -4.90154 \\ \mathrm{H} & -3.70184 & 0.67928 & -3.95067 \\ \mathrm{C} & -0.11157 & 2.48369 & -2.07303 \\ \mathrm{H} & 0.03654 & 2.65568 & -1.00965 \\ \mathrm{H} & -0.08702 & 3.44827 & -2.58928 \\ \mathrm{C} & 1.32181 & 1.33968 & -3.82832 \\ \mathrm{H} & 0.81911 & 1.77393 & -4.6778 \\ \mathrm{C} & 2.35182 & 0.46142 & -3.76223 \\ \mathrm{H} & 2.91422 & -0.02476 & -4.54345 \\ \mathrm{C} & 3.73489 & -0.56377 & -1.92207 \\ \mathrm{H} & 4.55486 & 0.07121 & -1.57676 \\ \mathrm{H} & 4.08782 & -1.19627 & -2.73654 \\ \mathrm{H} & 3.37372 & -1.19604 & -1.10753 \\ \mathrm{C} & 1.80021 & 0.99502 & -1.66269\end{array}$

$\begin{array}{cccc}\mathrm{C} & 1.73246 & 1.09387 & -0.21031 \\ \mathrm{H} & 1.77587 & 2.1553 & 0.06239 \\ \mathrm{H} & 2.60629 & 0.60878 & 0.22671 \\ \mathrm{C} & -1.23052 & -0.2027 & 2.57922 \\ \mathrm{C} & 0.03988 & 0.3719 & 3.01953 \\ \mathrm{C} & 0.13203 & 1.69157 & 2.50624 \\ \mathrm{C} & -1.05582 & 1.96061 & 1.70973 \\ \mathrm{C} & -1.9084 & 0.78665 & 1.80747 \\ \mathrm{C} & -1.76496 & -1.52779 & 3.01728 \\ \mathrm{H} & -0.99087 & -2.29845 & 2.97639 \\ \mathrm{H} & -2.59681 & -1.85415 & 2.38911 \\ \mathrm{H} & -2.13096 & -1.46829 & 4.04967 \\ \mathrm{C} & 0.98788 & -0.28218 & 3.97124 \\ \mathrm{H} & 0.62929 & -0.1487 & 4.99946 \\ \mathrm{H} & 1.98939 & 0.14813 & 3.9072 \\ \mathrm{H} & 1.07528 & -1.35282 & 3.77537 \\ \mathrm{C} & 1.21034 & 2.68274 & 2.80897 \\ \mathrm{H} & 2.16757 & 2.19449 & 3.00397 \\ \mathrm{H} & 0.94633 & 3.26329 & 3.70103 \\ \mathrm{H} & 1.35518 & 3.39589 & 1.99286 \\ \mathrm{C} & -1.47771 & 3.31595 & 1.23297 \\ \mathrm{H} & -2.22832 & 3.25692 & 0.44017 \\ \mathrm{H} & -0.63346 & 3.90505 & 0.86225 \\ \mathrm{H} & -1.9273 & 3.88764 & 2.05482 \\ \mathrm{C} & -3.29967 & 0.67793 & 1.2689 \\ \mathrm{H} & -4.0221 & 1.01382 & 2.02222 \\ \mathrm{H} & -3.54906 & -0.35151 & 1.00231 \\ \mathrm{H} & -3.43943 & 1.29907 & 0.38041 \\ \mathrm{C} & -1.47681 & 1.83032 & -2.30089 \\ \mathrm{H} & -1.79059 & 1.98896 & -3.33443 \\ \mathrm{H} & -2.20691 & 2.34074 & -1.66415\end{array}$

\section{IrIvyvyEt $: \mathrm{E}=-1192.73036649$}

\begin{tabular}{|c|c|c|c|}
\hline Ir & 0.10078 & 0.26082 & 0.58868 \\
\hline I & 0.01363 & -2.48027 & 0.85397 \\
\hline $\mathrm{N}$ & 1.38371 & 0.67138 & -2.15395 \\
\hline C & 0.26579 & 2.32677 & 1.30837 \\
\hline $\mathrm{C}$ & 1.47146 & 1.62831 & 1.7228 \\
\hline $\mathrm{C}$ & 1.09931 & 0.57093 & 2.59573 \\
\hline C & 0.22058 & 3.64435 & 0.5975 \\
\hline $\mathrm{H}$ & -0.72372 & 3.78538 & 0.06405 \\
\hline $\mathrm{H}$ & 1.03913 & 3.74906 & -0.12024 \\
\hline $\mathrm{H}$ & 0.31409 & 4.47156 & 1.31268 \\
\hline C & 2.86622 & 2.01275 & 1.34409 \\
\hline $\mathrm{H}$ & 3.53313 & 1.14824 & 1.32718 \\
\hline $\mathrm{H}$ & 3.26759 & 2.73084 & 2.06905 \\
\hline $\mathrm{H}$ & 2.90273 & 2.48326 & 0.35834 \\
\hline C & 2.01417 & -0.35456 & 3.33111 \\
\hline $\mathrm{H}$ & 2.13224 & -0.02181 & 4.3696 \\
\hline $\mathrm{H}$ & 3.00696 & -0.38327 & 2.87679 \\
\hline $\mathrm{H}$ & 1.62217 & -1.37492 & 3.34146 \\
\hline C & 1.38952 & -0.02223 & -0.93749 \\
\hline C & 2.42348 & 0.31582 & -2.94279 \\
\hline C & 0.32636 & 1.58936 & -2.52777 \\
\hline $\mathrm{H}$ & 0.68696 & 2.23125 & -3.33505 \\
\hline $\mathrm{H}$ & 0.10459 & 2.21263 & -1.66007 \\
\hline N & -1.82333 & 0.38529 & -1.96452 \\
\hline C & -0.84556 & 1.72623 & 2.03082 \\
\hline C & -0.34965 & 0.63824 & 2.79298 \\
\hline C & -2.2461 & 2.24908 & 2.02787 \\
\hline $\mathrm{H}$ & -2.97113 & 1.47402 & 2.28391 \\
\hline $\mathrm{H}$ & -2.34594 & 3.05245 & 2.76766 \\
\hline $\mathrm{H}$ & -2.52276 & 2.65871 & 1.05322 \\
\hline $\mathrm{C}$ & -1.1128 & -0.21523 & 3.75344 \\
\hline
\end{tabular}




$\begin{array}{lccl}\mathrm{H} & -0.93407 & 0.12348 & 4.78134 \\ \mathrm{H} & -2.18901 & -0.16595 & 3.57302 \\ \mathrm{H} & -0.80807 & -1.26257 & 3.68244 \\ \mathrm{C} & -1.53989 & 0.07069 & -0.6193 \\ \mathrm{C} & -3.10292 & 0.0685 & -2.28664 \\ \mathrm{~N} & 3.12156 & -0.59951 & -2.25775 \\ \mathrm{~N} & -3.67097 & -0.43413 & -1.18714 \\ \mathrm{C} & 2.50817 & -0.81755 & -1.03661 \\ \mathrm{C} & -2.74017 & -0.43455 & -0.17004 \\ \mathrm{H} & 2.8864 & -1.55294 & -0.34467 \\ \mathrm{H} & -2.97384 & -0.86078 & 0.79203 \\ \mathrm{C} & -5.04233 & -0.90893 & -1.06258 \\ \mathrm{H} & -5.74997 & -0.08069 & -1.14822 \\ \mathrm{H} & -5.25858 & -1.66162 & -1.8234 \\ \mathrm{H} & -5.15339 & -1.36958 & -0.08164 \\ \mathrm{C} & 4.33739 & -1.26549 & -2.70644 \\ \mathrm{H} & 4.16989 & -1.77207 & -3.65927 \\ \mathrm{H} & 5.15829 & -0.55107 & -2.80537 \\ \mathrm{H} & 4.6089 & -2.01215 & -1.9611 \\ \mathrm{C} & -3.75152 & 0.2434 & -3.61154 \\ \mathrm{H} & -3.79805 & 1.29821 & -3.90704 \\ \mathrm{H} & -3.21803 & -0.30162 & -4.39781 \\ \mathrm{H} & -4.77531 & -0.13209 & -3.5881 \\ \mathrm{C} & 2.74543 & 0.84475 & -4.29398 \\ \mathrm{H} & 1.96614 & 0.60377 & -5.02531 \\ \mathrm{H} & 2.87085 & 1.93295 & -4.28036 \\ \mathrm{H} & 3.67951 & 0.41525 & -4.65895 \\ \mathrm{C} & -0.92833 & 0.87688 & -3.01263 \\ \mathrm{H} & -0.64116 & 0.03639 & -3.65614 \\ \mathrm{H} & -1.49742 & 1.58085 & -3.62706\end{array}$

\section{bisImEt $^{2+}: \mathrm{E}=-687.795312231$}

$\mathrm{N}, 0,-0.9394908505,-0.0255146274,-3.0280946137$ C, $0,-0.5575953015,1.8137613895,-1.8769025612$ $\mathrm{N}, 0,0.3925105522,0.8573380392,-1.5599932498$ $\mathrm{C}, 0,-1.392303111,1.2555928783,-2.7902250442$ $\mathrm{N}, 0,0.8660079141,0.2797828827,1.5793375474$ C, $0,-1.5485660619,-0.9620198347,-3.9810349556$ $\mathrm{H}, \mathrm{O},-0.8294288222,-1.2272544923,-4.7578185724$ $\mathrm{H}, \mathrm{O},-2.4017918817,-0.4687226355,-4.4446059474$ $\mathrm{H}, \mathrm{O},-1.8967076988,-1.8569554901,-3.4623114001$ $\mathrm{C}, 0,0.1515837242,-0.260867373,-2.2807001617$ C, $0,0.9640647636,-1.5032566018,-2.2917193758$ $\mathrm{H}, \mathrm{O}, 0.4190808296,-2.313577925,-2.7776267389$ $\mathrm{H}, 0,1.2067741356,-1.8319366808,-1.2781675509$ $\mathrm{H}, 0,1.8999860206,-1.3567764616,-2.8436877642$ C, $0,-0.278040111,0.1495239353,2.2880885155$ C, $0,1.7059172267,-0.7803325166,1.8757558007$ $\mathrm{H}, \mathrm{O},-2.2544632751,1.6633946924,-3.296104025$ C, $0,-1.4197717065,1.0982868406,2.310902164$ $\mathrm{H}, \mathrm{O},-1.1440307262,2.0352159367,2.8084879812$ $\mathrm{H}, 0,-2.2600404583,0.6721429452,2.859869065$ $\mathrm{N}, 0,-0.1746580159,-0.9808604826,3.0064703553$ C, $0,1.0489112657,-1.5697526418,2.7632329017$ $\mathrm{H}, 0,2.7014042498,-0.8688177856,1.4681088465$ $\mathrm{H}, \mathrm{O}, 1.3522146424,-2.4851679187,3.2484556282$ C, $0,-1.1794962284,-1.5033403174,3.9413677852$ $\mathrm{H}, 0,-1.3305423918,-0.7992755004,4.7615612295$ $\mathrm{H}, \mathrm{O},-2.120381194,-1.6898662073,3.4209672275$ $\mathrm{H}, \mathrm{O},-0.8099323962,-2.4439483794,4.3473703666$ C, $0,1.2489728783,1.4297913233,0.7547200201$ $\mathrm{H}, 0,0.462229064,2.1818580592,0.8327906486$ $\mathrm{H}, 0,2.1503002751,1.8682366795,1.1947540577$ C, $0,1.5611303081,1.1074696818,-0.7107347729$
$\mathrm{H}, 0,2.0963147947,1.9656684197,-1.1292842972$ $\mathrm{H}, 0,2.2316433914,0.2504524759,-0.7913131316$ $\mathrm{H}, 0,-0.5430099013,2.8105623775,-1.463036519$ $\mathrm{H}, \mathrm{O},-1.769051735,1.3304066265,1.3010487275$

\section{bisImEtme $^{+}: \mathrm{E}=-687.304402473$}

$\mathrm{N}, 0,-1.2342565618,-0.2087957494,-2.6249425355$ C, $0,-0.2323371537,-1.1990511706,-0.9311295021$ $\mathrm{N}, 0,0.5480651233,-0.1597828111,-1.3965265274$ C, $0,-1.348329607,-1.2314785643,-1.704760025$ $\mathrm{N}, 0,0.6920936589,0.7284246865,1.3775136003$ C, $0,-2.2030937154,0.1017640373,-3.6718198329$ $\mathrm{H}, \mathrm{O},-2.4733347885,1.1587815726,-3.6344148044$ $\mathrm{H}, \mathrm{O},-3.0980551317,-0.49567664,-3.5027498303$ $\mathrm{H}, \mathrm{O},-1.7933457094,-0.1406691175,-4.6552092038$ C, $0,-0.0727321101,0.4412079758,-2.427274257$ C, $0,0.4018860315,1.5971159349,-3.227251009$ $\mathrm{H}, 0,-0.2512659755,2.4654958625,-3.0885797163$ $\mathrm{H}, 0,0.4156853388,1.3541198897,-4.2943799238$ $\mathrm{H}, \mathrm{O}, 1.4113495475,1.8889933082,-2.9380557128$ C, $0,0.7743567256,-0.4367994474,2.1399688724$ C, $0,-0.4608316672,1.4459105601,1.733282759$ $\mathrm{H}, \mathrm{O},-2.2052010245,-1.8860527096,-1.6822060525$ C, $0,1.7295583541,-1.4094419861,2.0876354201$ $\mathrm{H}, 0,2.6664650273,-1.2446846068,1.5725236518$ $\mathrm{H}, 0,1.6875789995,-2.246574891,2.7706523419$ $\mathrm{N}, 0,-0.365284787,-0.4194561679,2.9270277379$ C, $0,-1.0993101042,0.7358791023,2.681402881$ $\mathrm{H}, \mathrm{O},-0.6955074002,2.4011501137,1.2915550993$ $\mathrm{H}, \mathrm{O},-2.0119207809,0.9494206903,3.2153762165$ C, $0,-0.6419587324,-1.4207321717,3.9271729683$ $\mathrm{H}, \mathrm{O},-0.7763878588,-2.4073066738,3.4684519761$ $\mathrm{H}, 0,0.1795030675,-1.4828654646,4.6500148545$ $\mathrm{H}, 0,-1.5570741859,-1.1540491553,4.4572906982$ C, $0,1.6753016398,1.1465622937,0.4232779036$ $\mathrm{H}, 0,1.4022662871,2.1477640515,0.0782178764$ $\mathrm{H}, 0,2.6678133991,1.2233756609,0.8892752475$ $\mathrm{C}, 0,1.8266417121,0.1948327279,-0.7799452339$ $\mathrm{H}, 0,2.4826444191,0.6376061913,-1.5316804082$ $\mathrm{H}, 0,2.2719995199,-0.7479597581,-0.4606994509$ $\mathrm{H}, 0,0.0975569775,-1.7982137826,-0.0902544536$

\section{bisImEtvy $^{+}: \mathrm{E}=-687.287158225$}

$\mathrm{N}, 0,-0.9346164177,0.0200659293,-3.0662232471$ C, $0,-0.7726528437,0.8894969404,-0.9595495574$ $\mathrm{N}, 0,0.4329421962,0.3545766922,-1.4418741703$ C, $0,-1.6103293155,0.6502616046,-2.0246583844$ $\mathrm{N}, 0,0.9069695417,0.1453829006,1.6663629593$ C, $0,-1.5114822921,-0.3658960357,-4.3454557032$ $\mathrm{H}, \mathrm{O},-1.0206498198,0.1582644637,-5.1692451943$ $\mathrm{H}, \mathrm{O},-2.5659595633,-0.0907516127,-4.3385104224$ $\mathrm{H}, \mathrm{O},-1.4345624836,-1.4451369042,-4.4996028016$ C, $0,0.3382720297,-0.1581163886,-2.6911180231$ C, $0,1.4289968447,-0.7735159585,-3.4909774606$ $\mathrm{H}, \mathrm{O}, 1.0515976654,-1.1510205749,-4.4426746264$ $\mathrm{H}, 0,1.8885093092,-1.6164367802,-2.962519862$ $\mathrm{H}, 0,2.2222440338,-0.0503670285,-3.71482874$ C, $0,-0.2421737874,0.3934182325,2.3267950342$ C, $0,1.4508526202,-1.0401302108,2.1238788567$ $\mathrm{H}, \mathrm{O},-2.6595081182,0.8805168357,-2.1448726125$ C, $0,-1.1366674973,1.5374467803,2.0873629589$ $\mathrm{H}, 0,-1.2741079595,1.5623375998,0.9755320799$ $\mathrm{H}, \mathrm{0},-2.0903988278,1.4104401249,2.5999575119$ $\mathrm{H}, \mathrm{O},-0.6839283183,2.4781714786,2.4185567656$ 
$\mathrm{N}, 0,-0.43478374,-0.6307461492,3.1803067785$ C, $0,0.6067248976,-1.529737978,3.0658425387$ $\mathrm{H}, 0,2.3887631199,-1.4251314107,1.7566499156$ $\mathrm{H}, 0,0.6598109818,-2.4252405279,3.664505104$ C, $0,-1.5760199609,-0.7797295063,4.0783752841$ $\mathrm{H}, \mathrm{O},-1.7028887268,0.1228480267,4.6781767587$ $\mathrm{H}, 0,-2.4864504313,-0.9788650374,3.5089149466$ $\mathrm{H}, 0,-1.3815092324,-1.6198224728,4.7442662191$ C, $0,1.5446585015,1.0163807926,0.6727595736$ $\mathrm{H}, 0,0.9499637606,1.9256835781,0.5929360744$ $\mathrm{H}, 0,2.5407347324,1.2660265293,1.0518512184$ C, $0,1.6915843679,0.3918548636,-0.7093602093$ $\mathrm{H}, \mathrm{O}, 2.4208551682,1.0011493622,-1.2573238733$ $\mathrm{H}, 0,2.1133222614,-0.6180200779,-0.6351441535$

\section{bisImEtvycycTS $^{+}: \mathrm{E}=-687.281839805$}

$\begin{array}{cccc}\text { N } & -2.87923 & -0.96425 & -0.17902 \\ \mathrm{C} & -0.63423 & -0.73126 & 0.20191 \\ \mathrm{~N} & -1.3221 & 0.49203 & 0.08617 \\ \mathrm{C} & -1.66916 & -1.62289 & 0.03315 \\ \mathrm{~N} & 1.71817 & 1.05568 & 0.26134 \\ \mathrm{C} & -4.1704 & -1.60229 & -0.38765 \\ \mathrm{H} & -4.86532 & -1.35336 & 0.4184 \\ \mathrm{H} & -4.01831 & -2.68146 & -0.39439 \\ \mathrm{H} & -4.60157 & -1.30685 & -1.34738 \\ \mathrm{C} & -2.64983 & 0.35374 & -0.14505 \\ \mathrm{C} & -3.65255 & 1.43742 & -0.32053 \\ \mathrm{H} & -4.64008 & 1.02351 & -0.53054 \\ \mathrm{H} & -3.38932 & 2.09911 & -1.15306 \\ \mathrm{H} & -3.74249 & 2.05486 & 0.58106 \\ \mathrm{C} & 2.1394 & -0.18666 & 0.57036 \\ \mathrm{C} & 2.24306 & 1.42428 & -0.96258 \\ \mathrm{H} & -1.66228 & -2.70398 & 0.04168 \\ \mathrm{C} & 1.96219 & -0.94434 & 1.83133 \\ \mathrm{H} & 1.17542 & -0.51476 & 2.44546 \\ \mathrm{H} & 1.65657 & -1.96728 & 1.60712 \\ \mathrm{H} & 2.90208 & -0.95772 & 2.39858 \\ \mathrm{~N} & 2.96118 & -0.5795 & -0.41762 \\ \mathrm{C} & 3.02152 & 0.40121 & -1.39011 \\ \mathrm{H} & 2.02822 & 2.37851 & -1.4167 \\ \mathrm{H} & 3.6205 & 0.29102 & -2.27986 \\ \mathrm{C} & 3.62933 & -1.87302 & -0.48212 \\ \mathrm{H} & 4.19431 & -2.05503 & 0.43383 \\ \mathrm{H} & 2.89296 & -2.66711 & -0.62741 \\ \mathrm{H} & 4.3201 & -1.86544 & -1.32454 \\ \mathrm{C} & 0.64034 & 1.78016 & 0.92998 \\ \mathrm{H} & 0.48857 & 1.34139 & 1.91519 \\ \mathrm{H} & 0.97234 & 2.81311 & 1.07122 \\ \mathrm{C} & -0.66939 & 1.79116 & 0.14432 \\ \mathrm{H} & -1.33036 & 2.51155 & 0.63815 \\ \mathrm{H} & -0.48926 & 2.16717 & -0.87103\end{array}$

\section{$\mathbf{A}^{+}: \mathrm{E}=-687.313824187$}

$\begin{array}{cccc}\mathrm{N} & 1.04749 & -0.03635 & 2.2271 \\ \mathrm{C} & 0.36806 & 0.30274 & 0.14023 \\ \mathrm{~N} & -0.70517 & 0.00184 & 0.96278 \\ \mathrm{C} & 1.46607 & 0.28591 & 0.95182 \\ \mathrm{~N} & -1.21784 & 0.45986 & -1.75392 \\ \mathrm{C} & 1.92271 & -0.17432 & 3.38894 \\ \mathrm{H} & 1.609 & 0.50475 & 4.18378 \\ \mathrm{H} & 2.93642 & 0.08488 & 3.08611 \\ \mathrm{H} & 1.9158 & -1.2044 & 3.75201 \\ \mathrm{C} & -0.28486 & -0.20793 & 2.22153 \\ \mathrm{C} & -1.14215 & -0.53893 & 3.38649\end{array}$

$\begin{array}{lccl}\text { H } & -0.54617 & -0.95634 & 4.19942 \\ \mathrm{H} & -1.90009 & -1.27743 & 3.11323 \\ \mathrm{H} & -1.65575 & 0.35105 & 3.76863 \\ \mathrm{C} & 0.20316 & 0.58242 & -1.37363 \\ \mathrm{C} & -1.38104 & -0.83673 & -2.326 \\ \mathrm{H} & 2.50484 & 0.47763 & 0.73601 \\ \mathrm{C} & 0.75763 & 1.97076 & -1.6929 \\ \mathrm{H} & 0.22701 & 2.74468 & -1.1303 \\ \mathrm{H} & 1.81981 & 2.04633 & -1.4405 \\ \mathrm{H} & 0.63176 & 2.15378 & -2.76103 \\ \mathrm{~N} & 0.82731 & -0.45705 & -2.20101 \\ \mathrm{C} & -0.17873 & -1.35775 & -2.58498 \\ \mathrm{H} & -2.3583 & -1.17732 & -2.63643 \\ \mathrm{H} & 0.07167 & -2.2565 & -3.13059 \\ \mathrm{C} & 2.17458 & -0.91161 & -1.95426 \\ \mathrm{H} & 2.86349 & -0.06172 & -1.93038 \\ \mathrm{H} & 2.2912 & -1.49229 & -1.0243 \\ \mathrm{H} & 2.48468 & -1.54641 & -2.78792 \\ \mathrm{C} & -2.17939 & 0.8631 & -0.75135 \\ \mathrm{H} & -2.01476 & 1.91388 & -0.48831 \\ \mathrm{H} & -3.18551 & 0.79301 & -1.17392 \\ \mathrm{C} & -2.0992 & -0.01075 & 0.50216 \\ \mathrm{H} & -2.73901 & 0.36534 & 1.30399 \\ \mathrm{H} & -2.37964 & -1.04247 & 0.26768\end{array}$

\section{TSAB $: \mathrm{E}=-687.228489440$}

\section{$\mathrm{N}$}

C

$\mathrm{N}$

$\mathrm{C}$

$\mathrm{N}$

C

$\mathrm{H}$

$\mathrm{H}$

$\mathrm{H}$

C

C

$\mathrm{H}$

$\mathrm{H}$

$\mathrm{H}$

C

C

$\mathrm{H}$

C

H

$\mathrm{H}$
$\mathrm{H}$

N

$\mathrm{N}$
$\mathrm{C}$

C

$\mathrm{H}$
$\mathrm{C}$

C

$\mathrm{H}$
$\mathrm{H}$

$\mathrm{H}$
$\mathrm{H}$

$\mathrm{H}$

H

$\mathrm{H}$
$\mathrm{H}$

$\mathrm{H}$
$\mathrm{C}$

$\mathrm{H}$

$\mathrm{H}$
$\mathrm{H}$

0.19574

$-0.45643$

0.73047

$-0.88346$

0.71106

$-0.19656$

1.28173

1.31718

$-0.35096$

$-0.9183$

$-0.13976$

$-1.40832$

$-1.65448$

0.32444

$-0.54854$

1. 3062

0.67711

$-0.09155$

1.63323

0.76175

1.33407

0.77518

$-1.26516$

1. 36062

2.60514

3.07975

2. 49687

3. 25879

$-2.27096$

$-2.32975$

$-2.88351$

$-2.55871$

$-2.87029$

$-2.47967$

0.41439

2. 52104

0.37456

$-0.7239$

1.094

0.2098

0.7903

0.94598

1.71871

0.01368

$-0.67888$

$-1.66694$

$-2.11913$

$-2.463$

$-1.21074$

0.60886

$-0.69353$

2.00623

2.06483

2.74348

2.33957

2.18405

$-0.29436$

$-1.01063$

$-1.03541$

$-1.69194$

$-0.59004$

0.33145

$-1.2747$

$-1.04954$

0.49609

1.49685

0.48904

$-0.56993$

$-0.37577$

$-1.60245$

0.34054

0.82084

1.38163

$-1.90258$

3.88651

4.47492

3.87013

4.35999

2. 13918

3.09586

3.71972

2.53452

3.76727

$-1.14187$

$-2.87621$

1.42361

$-1.47539$

$-1.09654$

$-1.02177$

$-2.55731$

$-1.74499$

$-2.7489$

$-3.60824$

$-3.35116$

$-1.11947$

$-0.77355$

$-0.27064$

$-1.8636$

$-1.51435$

$-1.08044$

$-2.4263$

$-0.55811$

0.45801

$-0.87515$

\section{$\mathbf{B}^{+}: \mathrm{E}=-687.268638953$}

$\begin{array}{llll}\mathrm{N} & -2.6042 & -0.34436 & 0.50391\end{array}$ 


$\begin{array}{cccc}\mathrm{C} & -0.44173 & -0.23608 & 0.19372 \\ \mathrm{~N} & -1.0325 & 0.3504 & -0.90134 \\ \mathrm{C} & -1.4062 & -0.67202 & 1.0765 \\ \mathrm{~N} & 1.78362 & 0.97021 & 0.38414 \\ \mathrm{C} & -3.92428 & -0.62256 & 1.04879 \\ \mathrm{H} & -4.49771 & 0.30198 & 1.15069 \\ \mathrm{H} & -3.81593 & -1.07741 & 2.03343 \\ \mathrm{H} & -4.47017 & -1.3128 & 0.40039 \\ \mathrm{C} & -2.33189 & 0.27378 & -0.69159 \\ \mathrm{C} & -3.38882 & 0.77806 & -1.60954 \\ \mathrm{H} & -4.05189 & -0.02638 & -1.94616 \\ \mathrm{H} & -2.90928 & 1.21637 & -2.48504 \\ \mathrm{H} & -4.01229 & 1.54367 & -1.13459 \\ \mathrm{C} & 1.03596 & -0.38857 & 0.29995 \\ \mathrm{C} & 3.07113 & 0.73428 & -0.26104 \\ \mathrm{H} & -1.35568 & -1.18896 & 2.02327 \\ \mathrm{C} & 1.47053 & -1.20848 & 1.51853 \\ \mathrm{H} & 1.08609 & -0.78957 & 2.45136 \\ \mathrm{H} & 1.08548 & -2.22585 & 1.4229 \\ \mathrm{H} & 2.56023 & -1.25209 & 1.57536 \\ \mathrm{~N} & 1.66021 & -0.89852 & -0.92521 \\ \mathrm{C} & 2.89785 & -0.33586 & -1.05652 \\ \mathrm{H} & 3.86119 & 1.46386 & -0.19089 \\ \mathrm{H} & 3.61846 & -0.74056 & -1.75585 \\ \mathrm{C} & 1.25726 & -2.16354 & -1.50211 \\ \mathrm{H} & 1.48023 & -3.0229 & -0.85603 \\ \mathrm{H} & 0.18624 & -2.13922 & -1.71485 \\ \mathrm{H} & 1.78445 & -2.29601 & -2.44867 \\ \mathrm{C} & 1.59325 & 1.88335 & 1.52376 \\ \mathrm{H} & 0.85896 & 1.5459 & 2.24871 \\ \mathrm{H} & 2.50897 & 2.30019 & 1.92979 \\ \mathrm{C} & 1.11243 & 2.28572 & 0.18253 \\ \mathrm{H} & 0.05796 & 2.21291 & -0.05868 \\ \mathrm{H} & 1.70191 & 2.9743 & -0.41344 \\ & & & \end{array}$

TSBC $: \mathrm{E}=-687.225185444$

$\begin{array}{crcc}\mathrm{N} & -0.84068 & 0.44505 & -2.41626 \\ \mathrm{C} & -0.41455 & 0.1828 & -0.28742 \\ \mathrm{~N} & 0.08818 & -0.91845 & -0.93422 \\ \mathrm{C} & -1.00372 & 1.0369 & -1.19202 \\ \mathrm{~N} & 0.90803 & 0.9367 & 1.76928 \\ \mathrm{C} & -1.30212 & 0.96842 & -3.69277 \\ \mathrm{H} & -0.46162 & 1.1056 & -4.37771 \\ \mathrm{H} & -1.78212 & 1.93341 & -3.5302 \\ \mathrm{H} & -2.02702 & 0.2879 & -4.14672 \\ \mathrm{C} & -0.18274 & -0.74077 & -2.2136 \\ \mathrm{C} & 0.15729 & -1.68614 & -3.3116 \\ \mathrm{H} & -0.73879 & -2.05369 & -3.8237 \\ \mathrm{H} & 0.68487 & -2.54058 & -2.88686 \\ \mathrm{H} & 0.80195 & -1.22205 & -4.06604 \\ \mathrm{C} & -0.33042 & 0.33557 & 1.19926\end{array}$

$\begin{array}{lrcr}\mathrm{C} & 1.22393 & 0.21961 & 2.86204 \\ \mathrm{H} & -1.51143 & 1.98386 & -1.08657 \\ \mathrm{C} & -1.47148 & 1.234 & 1.73652 \\ \mathrm{H} & -1.37242 & 2.23707 & 1.31749 \\ \mathrm{H} & -2.43967 & 0.81956 & 1.44796 \\ \mathrm{H} & -1.41241 & 1.30919 & 2.82375 \\ \mathrm{~N} & -0.41096 & -0.95973 & 1.91238 \\ \mathrm{C} & 0.44422 & -0.93422 & 2.92704 \\ \mathrm{H} & 2.00983 & 0.5188 & 3.5428 \\ \mathrm{H} & 0.45898 & -1.71138 & 3.68174 \\ \mathrm{C} & -1.30867 & -2.04009 & 1.55304 \\ \mathrm{H} & -2.32014 & -1.65128 & 1.40379 \\ \mathrm{H} & -0.96342 & -2.50429 & 0.62597 \\ \mathrm{H} & -1.32309 & -2.77226 & 2.36141 \\ \mathrm{C} & 2.18279 & 1.57448 & 0.76662 \\ \mathrm{H} & 1.64837 & 2.30297 & 0.16326 \\ \mathrm{H} & 2.77424 & 2.03522 & 1.55611 \\ \mathrm{C} & 2.68463 & 0.43528 & 0.13022 \\ \mathrm{H} & 2.34929 & 0.13101 & -0.85143 \\ \mathrm{H} & 3.3376 & -0.24921 & 0.66277\end{array}$

\section{$\mathbf{C}^{+}: \mathrm{E}=-608.710508487$}

\begin{tabular}{|c|c|c|c|}
\hline $\mathrm{N}$ & -0.50586 & 0.47019 & 2.30664 \\
\hline $\mathrm{C}$ & -0.12883 & 0.37636 & 0.16562 \\
\hline N & 0.02149 & -0.89711 & 0.64261 \\
\hline $\mathrm{C}$ & -0.45861 & 1.2445 & 1.17661 \\
\hline $\mathrm{N}$ & -1.2719 & 0.32806 & -1.96409 \\
\hline C & -0.80319 & 0.9354 & 3.65288 \\
\hline $\mathrm{H}$ & -1.63093 & 0.36238 & 4.07696 \\
\hline $\mathrm{H}$ & -1.09161 & 1.98565 & 3.61186 \\
\hline $\mathrm{H}$ & 0.0727 & 0.83418 & 4.29916 \\
\hline C & -0.20523 & -0.81563 & 1.94173 \\
\hline C & -0.14457 & -1.94783 & 2.90483 \\
\hline $\mathrm{H}$ & 0.60861 & -1.78333 & 3.68317 \\
\hline $\mathrm{H}$ & 0.11314 & -2.85699 & 2.36113 \\
\hline $\mathrm{H}$ & -1.10711 & -2.10743 & 3.40304 \\
\hline $\mathrm{C}$ & -0.02282 & 0.64791 & -1.31419 \\
\hline $\mathrm{C}$ & -1.08641 & -0.67908 & -2.73885 \\
\hline $\mathrm{H}$ & -0.67319 & 2.30204 & 1.19989 \\
\hline $\mathrm{C}$ & 0.3858 & 2.08046 & -1.66772 \\
\hline $\mathrm{H}$ & -0.39666 & 2.7544 & -1.31623 \\
\hline $\mathrm{H}$ & 1.32641 & 2.34268 & -1.17932 \\
\hline $\mathrm{H}$ & 0.47843 & 2.20128 & -2.74954 \\
\hline N & 0.9482 & -0.34282 & -1.89762 \\
\hline C & 0.30657 & -1.11669 & -2.70478 \\
\hline $\mathrm{H}$ & -1.87075 & -1.13181 & -3.33652 \\
\hline $\mathrm{H}$ & 0.77924 & -1.93525 & -3.23803 \\
\hline $\mathrm{C}$ & 2.33251 & -0.46028 & -1.47334 \\
\hline $\mathrm{H}$ & 2.82464 & 0.51007 & -1.56258 \\
\hline $\mathrm{H}$ & 2.32758 & -0.782 & -0.42803 \\
\hline $\mathrm{H}$ & 2.84464 & -1.1949 & -2.09442 \\
\hline
\end{tabular}

